\title{
Multicomponent Composites, Electrical Networks and New Types of Continued Fraction I
}

\author{
G. W. Milton * \\ California Institute of Technology, 405-47, Pasadena, CA 91125, USA
}

\begin{abstract}
The development of bounds on the complex effective conductivity tensor $\sigma^{*}$ (that relates the average current to the average electric field in a multicomponent composite) has been hindered by lack of a suitable continuedfraction representation for $\sigma^{*}$. Here a new field equation recursion method is developed which gives an expression for $\sigma^{*}$ as a continued fraction of a novel form incorporating as coefficients the component conductivities and a set of fundamental geometric parameters reflecting the composite geometry. A hierarchy of field equations is set up such that the solutions of the $(j+1)$ thorder equation generate the solutions of the $j$ th-order equation. Consequently the effective tensor $\Omega^{(j)}$ associated with the $j$ th-order field equation is expressible as a fractional linear matrix transformation of $\Omega^{(j+1)}$. These transformations combine to form the continued fraction expansion for $\sigma^{*}=\Omega^{(0)}$ which is exploited in the following paper, Part II, to obtain bounds: crude bounds on $\Omega^{(j)}$, for $j \geqq 1$, give narrow bounds on $\sigma^{*}$. The continued fraction is a generalization to multivariate functions of the continued fraction expansion of single variable Stieltjes functions that proved important in the development of the theory of Páde approximants, asymptotic analysis, and the theory of orthogonal polynomials in the last century. The results extend to other transport problems, including conduction in polycrystalline media, the viscoelasticity of composites, and the response of multicomponent, multiterminal linear electrical networks.
\end{abstract}

\section{Introduction}

A central problem in Physics is the evaluation of the macroscopic response of a system given the formulae governing its microscopic behavior. Here a new field equation recursion method is introduced for estimating the effective transport

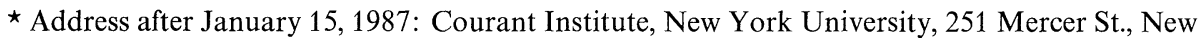
York, NY 10012, USA 
coefficients of composite materials. This development will likely have wide mathematical significance and open new areas for study in the analytic theory of continued fractions $[1,2]$ the analysis of functions of several complex variables and possibly in the field of multivariate Páde approximation [3].

Although the formalism set up in Sect. 2 extends to many transport problems, our prime focus is on estimating the effective conductivity tensor $\sigma^{*}$ of a macroscopically homogeneous $d$-dimensional composite constructed from $n$ isotropic components, or phases, with scalar conductivities $\sigma_{a}, a=1,2, \ldots, n$. Note that because of the standard mathematical analogies [4] all the results derived here apply to the effective diffusion constant, dielectric constant, thermal conductivity, and magnetic permeability of such composites. The extension of the results to composites with local anisotropy (i.e. polycrystalline media) and to the effective elasticity and viscoelasticity tensor follows from the work of Dell-Antoni et al. [5] and is not examined until Sect. 15. Similarly we defer until that section the consideration of the electrical response of multiterminal, multicomponent, linear impedance networks.

It is assumed the component phases are distinct and separated by sharp boundaries. In addition, the microstructure is required to be much larger than the atomic scale so the equations of classical physics can be employed. Examples of such composite materials include fluid-filled porous rocks, reinforced construction materials, colloidal suspensions, foams, fibrous thermal insulators, multiphase fluids, and granular aggregates.

The effective conductivity $\sigma^{*}$ is specifically defined as the tensor of proportionality relating the average current flow $\mathbf{j}$ in the composite to the average electric fields e via the equation

$$
\mathbf{j}=\sigma^{*} \mathbf{e} \text {. }
$$

In principle $\sigma^{*}$ can be computed by solving Laplace's equation, $\nabla^{2} \phi=0$, for the potential $\phi$ in each phase subject to the boundary conditions of continuity of both $\phi$ and the normal component of the current flow, $-\sigma \nabla \phi$, across each interface: the boundary conditions imposed on $\phi$ at infinity must ensure that the electric field $-\nabla \phi$ is on average uniform and equal to e. Accurate numerical calculations of $\sigma^{*}$ have thus been obtained for periodic arrays of spheres and lattices of cylinders of one component embedded in a matrix of a second component [6-9]. When the geometry is more complicated we need other methods for estimating $\sigma^{*}$. One successful approach has been to obtain bounds on $\sigma^{*}$ : a historical survey of work on estimating $\sigma^{*}$ through bounding techniques is given in the introduction to Part II, which follows this paper.

The field equation recursion method introduced here applies to composites having any given periodic microstructure. In various underlying respects it is a generalization of the Jacobi tridiagonalization method for deriving a continued fraction expansion for the leading diagonal coefficient $\widetilde{A}_{11}$ of the inverse $\tilde{A}$ of a given symmetric real matrix $A$ and thus is related to the method of minimal iterations (the Lanczos method) for finding the eigenvectors and eigenvalues of a matrix $[1,10]$. To understand the basic idea let us regard the elementary equation relating the local current field to the local electric field as a field equation in the Hilbert space $\mathscr{H}^{\prime}$ of square integrable fields. This Hilbert space contains a $d$ dimensional subspace $\mathscr{U}$ of uniform fields and various other subspaces $\mathscr{E}^{\prime}, \mathscr{J}^{\prime}$, and 
$\mathscr{P}_{a}^{\prime}, a=1,2, \ldots, n$, of curl-free electric fields, divergence-free current fields, and polarization fields that are non-zero only in component $a$.

The relevant fields in $\mathscr{H}^{\prime}$ can be ordered according to the sequence in which they appear in perturbation expansions of the solutions to the field equation in powers of the differences between the component conductivities in nearly homogeneous materials. The zeroth order fields are the uniform fields. Next come the first-order polarization fields that are uniform in one component and zero elsewhere. In general the $j$ th-order electric fields and current fields (or more correctly, the analogous electric displacement fields) are generated from the "surface charges" associated with the jth-order polarization fields. In turn the $(j+1)$ th-order polarization fields are obtained by taking each $j$ th-order electric field or current field and keeping it unchanged within one component while setting the field to zero elsewhere. The infinite hierarchy of fields thus defined spans some subspace $\mathscr{H} \equiv \mathscr{H}^{(0)} \subset \mathscr{H}^{\prime}$. The remaining fields in $\mathscr{H}^{\prime} \backslash \mathscr{H}$ are unimportant.

After some analysis we find it natural to introduce a hierarchy of subspaces $\mathscr{H}^{(0)} \supset \mathscr{H}^{(1)} \supset \mathscr{H}^{(2)} \ldots$, where $\mathscr{H}^{(j)}, j=1,2, \ldots, \infty$, is the orthogonal complement in $\mathscr{H}^{(0)}$ of the space spanned by the polarization fields of order $j$ or less. The relevant information about the composite microstructure is embodied in the inner products between the various fields and consequently these subspaces incorporate successively less of such information because they contain progressively fewer fields. The geometrical information contained in $\mathscr{H}^{(j-1)}$ but not in $\mathscr{H}^{(j)}$ is represented by a real $d(n-1)^{j}$-dimensional normalization matrix, $\mathcal{N}^{(j)}$, and by a set of $n$ real $d(n-1)^{j-1}$-dimensional weight matrices, $W_{a}^{(j-1)}, a=1,2, \ldots, n$, satisfying $\sum_{a=1}^{n} W_{a}^{(j-1)}=I^{(j-1)}$, where $I^{(j-1)}$ is the $d(n-1)^{j-1}$-dimensional identity matrix. The weights and normalization matrices are symmetric and positive-semidefinite and are defined in terms of the inner products between the jth-order fields in $\mathscr{H}^{(j-1)}$.

In essence the sequence of weights and normalization matrices describes the orientation of the three subspaces $\mathscr{U}, \mathscr{E}^{\prime}$, and $\mathscr{J}^{\prime}$, with respect to the $n$ subspaces $\mathscr{P}_{a}^{\prime}$, $a=1,2, \ldots, n$. Conversely, any such sequence of normalization and weight matrices describes the orientation in some Hilbert space $\mathscr{H}^{\prime}$ of three appropriately chosen orthogonal subspaces $\mathscr{U}, \mathscr{E}^{\prime \prime}$, and $\mathscr{J}^{\prime}$ spanning $\mathscr{H}^{\prime}$ with respect to $n$ other mutually orthogonal subspaces $\mathscr{P}_{a}^{\prime}, a=1,2, \ldots, n$, spanning $\mathscr{H}^{\prime}$. In a composite material the normalization and weight matrices can in principle be calculated from the $2 j$-point correlation functions characterizing the microstructure. The elementary weights satisfy $W_{a}^{(0)}=f_{a} l^{(0)}$, where $f_{a}$ is the volume fraction of component $a$ and when the composite is isotropic we find $N^{(1)}=(d-1) /^{(1)}$.

The lowest order fields in $\mathscr{H}^{(j)}$ span a $d(n-1)^{j}$-dimensional subspace $\mathscr{U}^{(j)}$. A field equation is easily set up in $\mathscr{H}^{(j)}$ that has exactly the same form as the original field equation in $\mathscr{H}^{(0)}$ : the associated "effective tensor," $\Omega^{(j)}$, maps $\mathscr{U}^{(j)}$ into $\mathscr{U}^{(j)}$. The key observation, made in Sect. 11, is that the solutions of the field equation in $\mathscr{H}^{(j)}$ generate all the solutions of the field equation in $\mathscr{H}^{(j-1)}$. This is remarkable because it allows us to obtain recursion relations that link together the effective tensors: we are able to express $\Omega^{(j-1)}$, as a fractional linear matrix transformation of $\Omega^{(j)}$, incorporating as coefficients the weights $W_{a}^{(j-1)}, a=1,2, \ldots, n$, the normalization factors $N^{(j)}$, and the component conductivities $\sigma_{a}, a=1,2, \ldots, n$. Since $\sigma^{*}=\Omega^{(0)}$, these fractional linear matrix transformations combine to form a 
continued fraction representation for $\sigma^{*}$, with matrices of increasing dimension as its elements. This is the basis of the field equation recursion method. The continued fractions, denoted as $\Omega$-functions, or $\Omega$-fractions, have some fascinating analytic and invariance properties which will be explored in Sect. 3 of Part II. They converge for any reasonable set of finite non-zero component conductivities: the dependence of $\sigma^{*}$ on $\Omega^{(j)}$ becomes negligible when $j>1$.

The continued fraction expansions are useful for two reasons. By truncating the continued fractions in an appropriate manner one obtains a sequence of rational approximants for the conductivity function $\sigma^{*}\left(\sigma_{1}, \sigma_{2}, \ldots, \sigma_{n}\right)$ each having the correct analytic form. The weights and normalization matrices that enter the truncated continued fraction can be adjusted to fit known values of the function and the rational approximant thus obtained can be used to extrapolate beyond the given data points. A second application of the continued fraction expansion is in deriving bounds on the effective conductivity tensor. These result from the observation that a crude estimate of $\Omega^{(j)}$, for $j \geqq 1$, generally gives a good estimate of $\sigma^{*}$ and wide bounds on $\Omega^{(j)}$ imply rather narrow bounds on $\sigma^{*}$. Thus we only need to obtain rather elementary bounds on $\Omega^{(j)}$ to give good bounds on $\sigma^{*}$. As will be seen in Part II, the field equation recursion method gives bounds on $\sigma^{*}$ for anisotropic composites constructed from arbitrarily many components which may have complex conductivities. Complex conductivities, or complex dielectric constants, are needed for a description of the response of the composite when the applied field $\mathbf{e}=\mathbf{e}(\omega)$ oscillates at a fixed frequency $\omega$ such that the wavelengths and attenuation lengths exceed the largest scale of inhomogeneities in the microstructure. At higher frequencies, outside this quasistatic regime, $\nabla \sigma \nabla \phi$ is non-zero on the length scale of the microstructure and scattering becomes important.

For two-component isotropic composites, various continued fraction expansions for $\sigma^{*}=\sigma^{*} /$ already exist. They were obtained solely on the basis of the analytic properties of $\sigma^{*}\left(\sigma_{1}, \sigma_{2}\right)$. Bergman [11] studied these analytic properties and from his work we now know $[12,13]$ that $\sigma^{*}\left(\sigma_{1}, \sigma_{2}\right) / \sigma_{1}=\sigma^{*}\left(1, \sigma_{2} / \sigma_{1}\right)$ is a Stieltjes function of $\sigma_{1} / \sigma_{2}$, analytic except on the negative real axis and satisfying $\sigma^{*} / \sigma_{1}>0$ when $\sigma_{1} / \sigma_{2}>0$. Stieltjes functions have been extensively studied in the mathematics literature and their continued fraction expansions are well-known: see for example Wall [1] and Jones and Thron [2]. Thus the continued fraction representation for $\sigma^{*}\left(\sigma_{1}, \sigma_{2}\right)$ obtained by Golden [14] (which was based on earlier work of Bergman [15]) is equivalent to the $S$-fraction expansion of a Stieltjes function. Another type of continued fraction representation for $\sigma^{*}\left(\sigma_{1}, \sigma_{2}\right)$, implicit in [12], has the appealing advantage of treating both components on a symmetric basis. It turns out that the field equation recursion method, for two-component isotropic composites, reproduces this expansion. We thereby gain an interpretation of the coefficients in the continued fraction in terms of overlaps between fields in the composite.

These continued fraction expansions for $\sigma^{*}$ were limited to two-component composites. Significant progress towards solving the multicomponent problem was made by Golden and Papanicolaou [16] who derived integral representations for the diagonal elements of $\sigma^{*}$. However, unlike the two-component case, this approach has not yet yielded any continued fraction expansions. Consequently the 
progress in deriving bounds on $\sigma^{*}\left(\sigma_{1}, \sigma_{2}, \ldots, \sigma_{n}\right)$ when the component conductivities are complex and when $n \geqq 3$ has been rather slow until now.

The outline of Part $I$ is as follows. Section 2 sets the problem in a simple mathematical framework. Perturbation expansions that serve to introduce the field hierarchies are developed in Sect. 3. From these fields the Gram-Schmidt orthogonalization is used in Sect. 4 to construct basis sets of fields in which electric fields of different orders, or current or polarization fields of different orders, are orthogonal but in which fields of the same order need not be orthogonal. Inner products between the various fields capture the relevant features of the composite geometry and define block-diagonal matrices of basic geometric parameters. In each basis the projection operators onto the relevant subspaces of $\mathscr{H}$ are represented by block-tridiagonal matrices of supplementary geometric parameters. Sections 5-7 explore relations between these geometric parameters and expressions are obtained for the supplementary geometric parameters in terms of the basic geometric parameters. The identities between the basic geometric parameters motivate us, in Sect. 8, to introduce a new basis set of fields for $\mathscr{H}$ : in fact we obtain a special set of orthonormal fields, denoted as a canonical basis set. From these fields we define the weights and normalization matrices. They are biased with respect to one of the components, called the reference medium, and determine the basic and supplementary geometric parameters. (A simpler, more direct, way of defining the canonical basis set of fields and the associated weights and normalization matrices is given in Appendix 1.) Section 9 shows that the two sets of weights and normalization factors associated with two different choices of reference medium are related by orthogonal transformations that in turn are determined by the lower order weights. In Sect. 10 we find that the duality of electric and current fields in two dimensions implies identities amongst the geometric parameters: the normalization factors of isotropic two-dimensional composites are simply identity matrices while anisotropic composites with $d=2$ and $n=2$ have isotropic weights and have normalization matrices of unit determinant.

The continued fraction expansions for $\sigma^{*}$ are developed in Sect. 11 first from variational principles and subsequently from the field equation recursion method. The recursion relations that generate these continued fractions are manipulated in Sect. 12 into other equivalent forms that generate other continued fraction expansions. Various types of terminating continued fractions are examined in Sect. 13 and the simplest of these correspond to well known variational bounds on $\sigma^{*}$ [17-22], denoted as Wiener-Beran and Hashin-Shtrikman variational bounds. Bruggeman's effective medium approximation [23] for $\sigma^{*}$ is found in Sect. 14 to be generated from a very simple choice of weights and normalization factors. Finally the extension of the results to related transport problems is discussed in Sect. 15.

In Part II, which follows directly after Part I, the analytic properties of the continued fractions are investigated and a comprehensive set of bounds on $\sigma^{*}$ is derived. When the component conductivities $\sigma_{a}, a=1,2, \ldots, n$, are complex, finite, and located in an open half of the complex plane it is found that each diagonal element of $\sigma^{*}$ is confined to a hierarchy of nested inclusion regions in the complex plane which converge to the exact value of the diagonal element as successively higher order weight matrices and normalization factors are incorporated in the 
bounds. The boundary of each region is piecewise analytic, comprised of at most $n$ circular arcs joined by sections which are not circular.

Those readers only interested in grasping the results should turn to Part II and read the Introduction and Sects. 2 and 4 in that part. Other readers who seek more details (but who don't want to follow the full treatment) should in addition read in Part I all of Sect. 2, the first few paragraphs in Sects. 3-8, and all of Appendix 1 and Sect. 11, and in Part II all of Sects. 3 and 4, and the first few paragraphs in Sect. 5-8.

The definitions given in Sect. 11 for the scalar weights $w_{1}^{(j)}=1-w_{2}^{(j)}$ and normalization factors $n^{(j)}$ of two-component isotropic composites differ slightly from the definitions of weights, $w_{1, j}=1-w_{2, j}$, and normalization factors, $n_{j}$, given earlier in [12]. They are, however, related via the identities

$$
w_{1}^{(j)} \equiv w_{1, j}, \quad w_{2}^{(j)} \equiv w_{2, j}, \quad n^{(j)} \equiv 1 / n_{j-1} .
$$

Also given any symmetric positive definite matrix $A$, such as $W_{a}^{(j)}$ or $N^{(j)}$, we will let

$$
\tilde{A}=A^{-1}, \quad \hat{A}=A^{1 / 2}, \quad \dot{A}=A^{-1 / 2},
$$

denote the inverse, square root and inverse square root of the matrix $A$ to avoid clashes between exponents and superscripts.

\section{Projection Operators and Hilbert Spaces}

This section is concerned with defining projection operators onto various Hilbert spaces associated with fields in composite materials. Similar formal approaches have been introduced by Willis [21], Kohler and Papanicolaou [24], Kantor and Bergman [25], and Dell-Antonio et al. [5]. The formalism leads to an elegant geometrical interpretation of the effective conductivity tensor $\sigma^{*}$.

Consider a $d$-dimensional periodic composite material with unit cell $\mathscr{D}$ of volume $\mathscr{V}(\mathscr{D})$ and let $\mathscr{H}^{\prime}$ denote the Hilbert space of square-integrable complex vector fields that have the same periodicity as the composite. On this Hilbert space the standard norm

$$
\left\langle\mathbf{P} \mid \mathbf{P}^{\prime}\right\rangle \equiv \frac{1}{\mathscr{V}(\mathscr{D})} \int_{\mathscr{D}} d \mathbf{x} \overline{\mathbf{P}}(\mathbf{x})^{T} \mathbf{P}^{\prime}(\mathbf{x})
$$

is defined for any two fields $\mathbf{P}, \mathbf{P}^{\prime} \in \mathscr{H}^{\prime}$, where $\overline{\mathbf{P}}$ denotes the complex conjugate of $\mathbf{P}$.

Three subspaces of $\mathscr{H}^{\prime}$ are of prime importance: these are (i) the Hilbert space $\mathscr{U}$ of uniform fields, (ii) the Hilbert space $\mathscr{E}^{\prime}$ of curl-free, average zero (electric) fields, and (iii) the Hilbert space $\mathscr{J}^{\prime}$ of divergence-free, average-zero (current) fields. The subspaces are real-symmetric in the sense that if they contain a field $\mathbf{P}$ then they also contain its complex conjugate $\overline{\mathbf{P}}$. Associated with these Hilbert spaces are the projection operators $\Gamma_{0}, \Gamma_{1}$, and $\Gamma_{2}$ defined through the equations

$$
\begin{gathered}
\left|\mathbf{P}_{0}\right\rangle \equiv \Gamma_{0}|\mathbf{P}\rangle \quad \text { iff } \quad \mathbf{P}_{0}=\frac{1}{\mathscr{V}(\mathscr{D})} \int_{\mathscr{D}} d \mathbf{x P}(\mathbf{x}), \\
|\mathbf{E}\rangle \equiv \Gamma_{1}|\mathbf{P}\rangle \quad \text { iff } \quad \nabla \times \mathbf{E}=0, \quad \nabla \cdot(\mathbf{E}-\mathbf{P})=0, \quad \Gamma_{0}|\mathbf{E}\rangle=0, \\
|\mathbf{J}\rangle \equiv \Gamma_{2}|\mathbf{P}\rangle \quad \text { iff } \quad \nabla \cdot \mathbf{J}=0, \quad \nabla \times(\mathbf{J}-\mathbf{P})=0, \quad \Gamma_{0}|\mathbf{J}\rangle=0 .
\end{gathered}
$$


By the divergence theorem, it follows that $\Gamma_{0}, \Gamma_{1}$, and $\Gamma_{2}$ project onto mutually orthogonal spaces $\left(\mathscr{U}, \mathscr{E}^{\prime}\right.$, and $\left.\mathscr{J}^{\prime}\right)$ and hence satisfy the identities

$$
\Gamma_{i} \Gamma_{j}=\delta_{i j} \Gamma_{i}
$$

Also, since any average-zero vector field can be split into divergence-free and curlfree parts, we have

$$
\sum_{i} \Gamma_{i}=I
$$

in which $I$ is the identity operator. Because of this last identity it is only necessary to introduce the operators $\Gamma_{0}$ and $\Gamma_{1}$, and $\Gamma_{1}$ is in fact just the integral operator $\nabla \Delta^{-1} \nabla$, where $\Delta^{-1}$ is the inverse Laplacian [24]. (In Fourier $\mathbf{k}$-space the operator $\Gamma_{1}$ is local with elements $k_{i} k_{j} / k^{2}$ when $\mathbf{k} \neq 0$ and with zero elements when $\mathbf{k}=0$.) To preserve symmetry, we will also use the operator $\Gamma_{2}$.

The composite is assumed to be composed of a finite number, $n$, of components, each with homogeneous, isotropic conductivity $\sigma_{a}, a=1,2, \ldots, n$. The structure of the composite is represented by the characteristic functions,

$$
\begin{aligned}
& \chi_{a}=1 \quad \text { in component } a ; \\
& \chi_{a}=0 \text { elsewhere, }
\end{aligned}
$$

which can each be regarded as a projection operator mapping any field $\mathbf{P} \in \mathscr{H}^{\prime}$ onto the real-symmetric Hilbert space $\mathscr{P}_{a}^{\prime}$ consisting of all those fields which vanish outside component $a$. Since no two components overlap, these operators satisfy

$$
\chi_{a} \chi_{b}=\delta_{a b} \chi_{a},
$$

and because the components occupy all space we have

$$
\sum_{a=1}^{n} \chi_{a}=I
$$

The operators $\chi_{a}$ and $\Gamma_{i}$ do not commute.

In terms of these operators $\chi_{a}$, the local conductivity, $\sigma$, of the composite is given by

$$
\sigma=\sum_{a=1}^{n} \chi_{a} \sigma_{a}
$$

and relates the equilibrium current field, $\mathbf{j}+\mathbf{J}^{*}(\mathbf{j})$, in the composite [where $\mathbf{j} \in \mathscr{U}$ and $\left.\mathbf{J}^{*}(\mathbf{j}) \in \mathscr{J}^{\prime}\right]$ to the equilibrium electric field, $\mathbf{e}+\mathbf{E}^{*}(\mathbf{e})\left[\right.$ where $\mathbf{e} \in \mathscr{U}$ and $\left.\mathbf{E}^{*}(\mathbf{e}) \in \mathscr{E}^{\prime}\right]$, via the field equation

$$
\left|\mathbf{j}+\mathbf{J}^{*}(\mathbf{j})\right\rangle=\sigma\left|\mathbf{e}+\mathbf{E}^{*}(\mathbf{e})\right\rangle .
$$

As Papanicolaou and Varadan [26] and Golden and Papanicolaou [27] have established on the basis of the Lax-Milgram lemma [28], this field equation has a unique solution for $\mathbf{j}, \mathbf{J}^{*}(\mathbf{j})$, and $\mathbf{E}^{*}(\mathbf{e})$ as a function of the average applied field $\mathbf{e}$ provided the $n$ component conductivities $\sigma_{a}, a=1,2, \ldots, n$, are finite and lie in an open half of the complex plane, and provided the characteristic functions are measurable. Under these conditions, (2.11) similarly has a unique solution for e, $\mathbf{E}^{*}(\mathbf{e})$, and $\mathbf{J}^{*}(\mathbf{j})$ as a function of $\mathbf{j}$. 
Once the field equation is solved the effective conductivity tensor $\sigma^{*}$ can easily be computed from its defining equation (1.1). The function $\sigma^{*}\left(\sigma_{1}, \sigma_{2}, \ldots, \sigma_{n}\right)$ thus obtained is merely determined by the orientation of the three orthogonal subspaces $\mathscr{U}, \mathscr{E}$, and $\mathscr{J}^{\prime}$ relative to the orthogonal family $\mathscr{P}_{a}^{\prime}, a=1,2, \ldots, n$. In the ensuing analysis of Sects. 3-8 we strive to obtain a concise way of representing the relative geometrical orientation of these subspaces.

\section{Perturbation Expansions and Field Hierarchies}

Perturbation expansions for electric fields and current fields within nearly homogeneous materials have been studied since the pioneering work of Brown [29]: see also [30, 31] among others. Here we show how the perturbation expansions can be naturally expressed in terms of hierarchies of electric fields and current fields: these real fields depend on the structure of the composite and not on the material properties of the constituents. The approach serves to introduce the field hierarchies, which form the foundation for developing a suitable basis set of fields that can be utilized to characterize the relative orientation of the subspaces and subsequently to obtain a continued fraction expansion for $\sigma^{*}$. We also define two hierarchies of polarization fields.

Let us first derive a perturbation expansion for the electric field $\mathbf{E}^{*}(\mathbf{e})$ for any applied field $\mathbf{e}$. Since $\mathbf{j}+\mathbf{J}^{*}$ is orthogonal to the space $\mathscr{E}^{\prime \prime}$ we have

$$
\Gamma_{1} \sigma\left|\mathbf{e}+\mathbf{E}^{*}(\mathbf{e})\right\rangle=0 .
$$

To solve this for $\mathbf{E}^{*}(\mathbf{e})$, choose one of the components, say component $q$, as a reference medium and define the conductivity differences

$$
\delta \sigma_{a}^{(q)} \equiv \sigma_{a}-\sigma_{q},
$$

which are small for a nearly homogeneous material. From (2.10) it follows that

$$
\sigma=\sigma_{q} I+\sum_{a=1}^{n} \chi_{a} \delta \sigma_{a}^{(q)}=\sigma_{q}\left(I-\sum_{a \neq q} \chi_{a} / s_{a}^{(q)}\right)
$$

where we have introduced the variables

$$
s_{a}^{(q)} \equiv-\sigma_{q} / \delta \sigma_{a}^{(q)}=\sigma_{q} /\left(\sigma_{q}-\sigma_{a}\right),
$$

as defined by Bergman [11]. Substituting (3.3) in (3.1) gives

$$
\left[I-\sum_{a \neq q} \Gamma_{1} \chi_{a} \mid s_{a}^{(q)}\right]\left|\mathbf{E}^{*}(\mathbf{e})\right\rangle=\sum_{a \neq q} \Gamma_{1} \chi_{a}|\mathbf{e}\rangle / s_{a}^{(a)},
$$

which can be solved by taking the inverse in $\mathscr{E}$ of the operator on the left-hand side and applying it to the right-hand side. Alternatively, to obtain a perturbation expansion (3.5) can be solved by iteration to yield the expansion

$$
\left|\mathbf{E}^{*}(\mathbf{e})\right\rangle=\sum_{a \neq q} \Gamma_{1} \chi_{a}|\mathbf{e}\rangle / s_{a}^{(q)}+\sum_{a, b \neq q} \Gamma_{1} \chi_{a} \Gamma_{1} \chi_{b}|\mathbf{e}\rangle / s_{a}^{(q)} s_{b}^{(q)}+\ldots
$$

for the fluctuating part of the electric field.

To simplify the form of this result we define the new variables

$$
s_{a b}^{(q)} \equiv s_{a}^{(q)} S_{b}^{(q)}, \quad S_{a \alpha}^{(q)} \equiv s_{a}^{(q)} S_{\alpha}^{(q)},
$$


where $\alpha=b, b c, b c d, \ldots$, represents a string of component indices, each taking values $1,2, \ldots, n$. Also we choose a basis set for $\mathscr{U}$ of orthonormal real-valued uniform fields $x_{\ell}, \ell=1,2, \ldots, d$, and define the hierarchy of real-valued electric fields

$$
\left|\mathbf{E}_{a \ell}^{0}\right\rangle \equiv \Gamma_{1} \chi_{a}\left|\mathbf{x}_{\ell}\right\rangle, \quad\left|\mathbf{E}_{a \tau}^{0}\right\rangle \equiv \Gamma_{1} \chi_{a}\left|\mathbf{E}_{\tau}^{0}\right\rangle,
$$

where $\tau=\alpha \ell=b \ell, b c \ell, \ldots$ represents a string, $\alpha$, of component indices followed by a single direction index $\ell$.

To avoid confusion, italic subscripts $(\neq i, j$ or $h)$ are reserved for component indices, script subscripts, such as $\ell, k$, and $m$, represent direction indices, and Greek subscripts stand for strings of indices: the subscripts $\alpha$ and $\beta$ signify strings of component indices while all other Greek subscripts represent a string of component indices followed by a direction index. We adopt the summation convention that sums over repeated Greek or script subscripts are implied, while sums over repeated italic subscripts are not implied. Given any string $\tau$ or $\alpha$ we let $o(\tau)$ and $o(\alpha)$ denote the number of component indices they contain, which we define as the order of the string.

With these definitions, the perturbation solution is simply

$$
\mathbf{E}^{*}(\mathbf{e})=\left\langle\mathbf{e} \mid \mathbf{x}_{\ell}\right\rangle \mathbf{E}_{\alpha \ell}^{0} / s_{\alpha}^{(q)} .
$$

Thus the electric field $\mathbf{E}^{*}$ can be expressed in terms of the fields $\mathbf{E}_{\tau}^{0}$.

A perturbation expansion for the current field $\mathbf{J} *(\mathbf{j})$ as a function of the applied current $\mathbf{j}$, is obtained by similar steps. We introduce Bergman's complementary variables

$$
t_{a}^{(q)} \equiv \sigma_{a} /\left(\sigma_{a}-\sigma_{q}\right)=1-s_{a}^{(q)}
$$

and define

$$
t_{a b}^{(q)} \equiv-t_{a}^{(q)} t_{b}^{(q)}, \quad t_{a \alpha}^{(q)} \equiv-t_{a}^{(q)} t_{\alpha}^{(q)},
$$

together with the hierarchy of real-valued current fields,

$$
\left|\mathbf{J}_{a \ell}^{0}\right\rangle=\Gamma_{2} \chi_{a}\left|\mathbf{x}_{\ell}\right\rangle, \quad\left|\mathbf{J}_{a \tau}^{0}\right\rangle \equiv-\Gamma_{2} \chi_{a}\left|\mathbf{J}_{\tau}^{0}\right\rangle
$$

in which the minus signs are introduced to simplify subsequent calculations. Starting from the field equation

$$
\Gamma_{2} \sigma^{-1}\left|\mathbf{j}+\mathbf{J}^{*}(\mathbf{j})\right\rangle=0
$$

the analysis yields the perturbation expansion

$$
\mathbf{J}^{*}(\mathbf{j})=\left\langle\mathbf{j} \mid \mathbf{x}_{\ell}\right\rangle \mathbf{J}_{\alpha \ell}^{0} / t_{\alpha}^{(q)}
$$

for the fluctuating part of the current field.

We have thus defined suitable hierarchies of electric fields and current fields. The definitions suggest we define a hierarchy of real-valued polarization fields, either via the recursion relations

$$
\left|\mathbf{P}_{a \ell}^{0}\right\rangle \equiv \chi_{a}\left|\mathbf{x}_{\ell}\right\rangle, \quad\left|\mathbf{P}_{a \tau}^{0}\right\rangle \equiv \chi_{a} \Gamma_{1}\left|\mathbf{P}_{\tau}^{0}\right\rangle,
$$

or through the complementary recursion relations

$$
\left|\mathbf{P}_{a \ell}^{00}\right\rangle \equiv \chi_{a}\left|\mathbf{x}_{\ell}\right\rangle, \quad\left|\mathbf{P}_{a \tau}^{00}\right\rangle \equiv-\chi_{a} \Gamma_{2}\left|\mathbf{P}_{\tau}^{00}\right\rangle .
$$


These two sets of polarization fields $\mathbf{P}_{a \tau}^{0}$ and $\mathbf{P}_{a \tau}^{00}$ are equally suitable for our analysis.

From the commutation relations (2.8) satisfied by the operators $\chi_{a}$ it immediately follows that

$$
\left\langle\mathbf{P}_{a \tau}^{0} \mid \mathbf{P}_{b w}^{0}\right\rangle=\left\langle\mathbf{P}_{a \tau}^{00} \mid \mathbf{P}_{b w}^{00}\right\rangle=0 \quad \text { if } \quad a \neq b .
$$

Likewise the commutation relations (2.5) for the operators $\Gamma_{i}$ imply

$$
\left\langle\mathbf{E}_{\tau}^{0} \mid \mathbf{J}_{\omega}^{0}\right\rangle=0 .
$$

The hierarchies of fields $\mathbf{E}_{\tau}^{0}, \mathbf{J}_{\tau}^{0}$, and $\mathbf{P}_{a \tau}^{0}$ (or $\mathbf{P}_{a \tau}^{00}$ ), with $\tau$ ranging over all possible strings span spaces $\mathscr{E}, \mathscr{J}$, and $\mathscr{P}_{a}$ that are subspaces of $\mathscr{E}^{\prime}, \mathscr{J}^{\prime}$, and $\mathscr{P}_{a}^{\prime}$. Since the LaxMilgram lemma [28] implies the field solutions $\mathbf{E}^{*}(\mathbf{e})$ and $\mathbf{J}^{*}(\mathbf{j})$ of (2.11) actually lie in the Hilbert spaces $\mathscr{E}$ and $\mathscr{J}$, there is no need to consider the larger spaces $\mathscr{E}^{\prime}, \mathscr{J}^{\prime}$ or $\mathscr{P}_{a}^{\prime}$.

\section{Block-Orthogonal Field Basis Sets}

The fields $\mathbf{E}_{\tau}^{0}, \mathbf{J}_{\tau}^{0}$, and $\mathbf{P}_{a \tau}^{0}$ (or $\mathbf{P}_{a \tau}^{00}$ ) defined in the previous section do not have any special orthogonality properties apart from (3.17) and (3.18). Hence they are unsuitable as basis sets of fields. In this section, following the spirit of the Jacobi tridiagonalization method [10], we use the Gram-Schmidt orthogonalization process to construct a new set of real fields $\mathbf{E}_{\tau}, \mathbf{J}_{\tau}$, and $\mathbf{P}_{a \tau}$ which are blockorthogonal to the extent that

$$
\left\langle\mathbf{E}_{\tau} \mid \mathbf{E}_{\mu}\right\rangle=\left\langle\mathbf{J}_{\tau} \mid \mathbf{J}_{\mu}\right\rangle=\left\langle\mathbf{P}_{a \tau} \mid \mathbf{P}_{a \mu}\right\rangle=0 \quad \text { whenever } \quad o(\mu) \neq o(\tau),
$$

where the order $o(\omega)$ denotes the number of component indices a string $\omega$ contains.

For each component index $a=1,2, \ldots, n$ and each integer $h \geqq 2$, let $\mathscr{E}_{h}^{<}, \mathscr{J}_{h}^{<}$, and $\mathscr{P}_{a, h}^{<}$denote the Hilbert spaces spanned by the sets of fields

$$
\bigcup_{\substack{\tau \\ o(\tau)<h}}\left\{\mathbf{E}_{\tau}^{0}\right\}, \quad \bigcup_{\substack{\tau \\ o(\tau)<h}}\left\{\mathbf{J}_{\tau}^{0}\right\}, \quad \bigcup_{\substack{\tau \\ o(a \tau)<h}}\left\{\mathbf{P}_{a \tau}^{0}\right\}
$$

respectively. To establish an appropriate basis set for these Hilbert spaces, first define the first-order fields,

$$
\mathbf{E}_{a \ell} \equiv \mathbf{E}_{a \ell}^{0}, \quad \mathbf{J}_{a \ell} \equiv \mathbf{J}_{a \ell}^{0}, \quad \mathbf{P}_{a \ell} \equiv \mathbf{P}_{a \ell}^{0}=\mathbf{P}_{a \ell}^{00},
$$

for all component indices, $a$, and direction indices, $\ell$. Next, for $h=2,3, \ldots, \infty$, and for all strings $\tau$ and $\omega$ with $o(\tau)=o(\omega)+1=h$ let the $h$ th-order fields $\mathbf{E}_{\tau}, \mathbf{J}_{\tau}$, and $\mathbf{P}_{a \omega}$ denote the components of $\mathbf{E}_{\tau}^{0}, \mathbf{J}_{\tau}^{0}$, and $\mathbf{P}_{a \omega}^{0}$ that are orthogonal to the Hilbert spaces $\mathscr{E}_{h}^{<}, \mathscr{J}_{h}^{<}$, and $\mathscr{P}_{a, h}^{<}$respectively. [The field $\mathbf{P}_{a \omega}$ can alternatively be defined as the component of $\mathbf{P}_{a \omega}^{00}$ that is orthogonal to $\mathscr{P}_{a, h}^{<}$: the equivalence of these two definitions follows from (2.6).] By this process of construction, the fields $\mathbf{E}_{\tau}, \mathbf{J}_{\tau}$, and $\mathbf{P}_{a \tau}$ are necessarily block-orthogonal.

Now from the summation relations (2.9) satisfied by the characteristic functions $\chi_{a}$ it follows that

$$
\sum_{a=1}^{n} \mathbf{E}_{a \tau}^{0}=\mathbf{E}_{\tau}^{0}, \quad \sum_{a=1}^{n} \mathbf{J}_{a \tau}^{0}=-\mathbf{J}_{\tau}^{0}
$$


which implies

$$
\sum_{a=1}^{n} \mathbf{E}_{a \tau}=0, \quad \sum_{a=1}^{n} \mathbf{J}_{a \tau}=0 .
$$

More generally, by the same reasoning given above, we have

$$
\sum_{b=1}^{n} \mathbf{E}_{\tau}=\sum_{b=1}^{n} \mathbf{J}_{\tau}=\sum_{b=1}^{n} \mathbf{P}_{a \tau}=0
$$

for any component index, $b$, in the string $\tau$.

Thus the fields $\mathbf{E}_{\tau}, \mathbf{J}_{\tau}$, and $\mathbf{P}_{a \tau}$ are not independent. To remove this degeneracy and obtain basis sets of (generally) independent real fields, we exclude those fields $\mathbf{E}_{\tau}, \mathbf{J}_{\tau}$, and $\mathbf{P}_{a \tau}$ for which $\tau$ contains a component index taking the value $q$. The fields in the remaining basis sets will be denoted by $\mathbf{E}_{\tau}^{(q)}, \mathbf{J}_{\tau}^{(q)}$, and $\mathbf{P}_{a \tau}^{(q)}$. Note that the index $a$ in $\mathbf{P}_{a \omega}^{(q)}$ can take the value $q$.

\section{The Basic and Supplementary Geometric Matrices}

Not all geometrical features of a composite are relevant in determining the effective conductivity: there is considerable degeneracy in the sense that many different composites share the same effective conductivity, even as the component conductivities are varied. Thus it is important to define a set of geometrical parameters, that characterize the relevant structural features of the composite. One candidate is the set of coefficients in the series expansion for $\sigma^{*}$ in terms of the differences $\delta \sigma_{a}^{(q)}$ between the component conductivities. This set, however, is unsuitable because it is difficult to calculate the constraints these coefficients must satisfy to give a sensible conductivity function with the right analytic properties. Golden and Papanicolaou [16], in a major advance, expressed the relevant structural aspects in terms of a positive measure $\mu$ that occurs in their integral representation formula for the effective conductivity. Unfortunately the measure must satisfy certain Fourier constraints that are hard to manipulate and lead to some unsolved problems in mathematics concerning the characterization of extremal measures of analytic functions [14].

Clearly the fields $\mathbf{E}_{\tau}, \mathbf{J}_{\tau}$, and $\mathbf{P}_{a \tau}$ contain a tremendous amount of geometric information. We now assert, and prove in Sect. 11, that the relevant geometric information is contained in the various real inner products,

$$
U_{\lambda, \eta} \equiv\left\langle\mathbf{E}_{\lambda} \mid \mathbf{E}_{\eta}\right\rangle, \quad V_{\lambda, \eta} \equiv\left\langle\mathbf{J}_{\lambda} \mid \mathbf{J}_{\eta}\right\rangle, \quad Z_{a, \lambda, \eta} \equiv\left\langle\mathbf{P}_{a \lambda} \mid \mathbf{P}_{a \eta}\right\rangle,
$$

which we denote as basic geometric matrices and in the real inner products,

$$
\begin{array}{ll}
U_{a, \lambda, \eta}^{\dagger} \equiv\left\langle\mathbf{E}_{\lambda}\left|\chi_{a}\right| \mathbf{E}_{\eta}\right\rangle, & V_{a, \lambda, \eta}^{\dagger} \equiv\left\langle\mathbf{J}_{\lambda}\left|\chi_{a}\right| \mathbf{J}_{\eta}\right\rangle, \\
Z_{1, \lambda, \eta}^{\dagger} \equiv\left\langle\mathbf{P}_{\lambda}\left|\Gamma_{1}\right| \mathbf{P}_{\eta}\right\rangle, & Z_{2, \lambda, \eta}^{\dagger} \equiv\left\langle\mathbf{P}_{\lambda}\left|\Gamma_{2}\right| \mathbf{P}_{\eta}\right\rangle,
\end{array}
$$

which we call supplementary geometric matrices. These real geometric matrices characterize the relative orientation of the $n$ orthogonal subspaces $\mathscr{P}_{a}, a=1,2, \ldots, n$ with respect to the three orthogonal subspaces $\mathscr{U}, \mathscr{E}$, and $\mathscr{J}$. We will see, however, that much of the information they contain is redundant. Our eventual aim is to obtain a minimal set of invariants (namely the weights and normalization 
matrices) that characterize the relative orientation of the subspaces and, most importantly, that satisfy simple constraints.

As discussed elsewhere [22, 29-31] geometric parameters such as these can be expressed as multiple integrals, with appropriate kernels, of the $h$-point correlation functions characterizing the geometry of the composite: $U_{\lambda, \eta}, V_{\lambda, \eta}, Z_{1, \lambda, \eta}^{\dagger}$, and $Z_{2, \lambda, \eta}^{\dagger}$ depend on $o(\lambda \eta)$-point correlation functions while $Z_{a, \lambda, \eta}, U_{a, \lambda, \eta}^{\dagger}$, and $V_{a, \lambda, \eta}^{\dagger}$ depend on $o(a \lambda \eta)$-point correlation functions. The $h$-point correlation functions each give the probability that a configuration of $h$ points placed randomly in the composite lands with certain points in component 1 , other specified points in component $2, \ldots$, and the remaining points in component $n$ : see Beran [31], Corson [32], Torquato and Stell [33], and Gillette [34] for a discussion of these correlation functions. The tremendous amount of statistical information contained in the $h$-point correlation functions currently makes their measurement prohibitive for $h>3$. Nevertheless, the automated measurement techniques of Berryman [35] and the Monte-Carlo computer simulations of Haile, Massobrio, and Torquato [36] hold great promise. Even if few of the geometrical parameters can be evaluated, the important point is that they are well-defined and lead to a continued fraction representation for the effective conductivity, from which a comprehensive set of bounds can be derived.

The geometric matrices have some obvious properties. By construction of the fields $\mathbf{E}_{\tau}, \mathbf{J}_{\tau}$, and $\mathbf{P}_{a \tau}$ [see (4.1)] the basic geometric parameters are block-diagonal in the sense that

$$
U_{\lambda, \eta}=V_{\lambda, \eta}=Z_{a, \lambda, \eta}=0 \quad \text { when } \quad o(\lambda) \neq o(\eta)
$$

In the special case where $o(\lambda)=o(\eta)=0$ the elements of $Z_{a, \lambda, \eta}$ can be directly evaluated from (3.15) to give

$$
Z_{a, \ell, k}=f_{a} \delta_{\ell k},
$$

where $f_{a}$ is the volume fraction of component $a$, satisfying

$$
\sum_{a=1}^{n} f_{a}=1
$$

Although not needed in subsequent analysis, we remark here that the elementary submatrices $U_{\lambda, \eta}$ and $V_{\lambda, \eta}$, with $o(\lambda)=o(\eta)=1$, have the additional property

$$
U_{a \ell, b \ell}=(d-1)^{-1} V_{a \ell, b \ell}=\delta_{a b} f_{a}-f_{a} f_{b},
$$

which directly follows from the observation that the trace of $\Gamma_{1}$, taken over the direction indices, acts as the projection operator onto the class of scalar functions with zero mean: see [37].

Now noting from (2.6) that the operators $\Gamma_{i}$ sum to the identity operator and recognizing that

$$
\left\langle\mathbf{P}_{a \lambda}\left|\Gamma_{0}\right| \mathbf{P}_{a \eta}\right\rangle=\delta_{\lambda \ell} \delta_{\ell \eta} f_{a} f_{b}=Z_{a, \lambda, \ell} Z_{b, \ell, \eta}
$$

[which vanishes unless $o(\lambda)=o(\eta)=0$ ], we easily deduce the identity

$$
Z_{1, a \lambda, b \eta}^{\dagger}+Z_{2, a \lambda, b \eta}^{\dagger}+Z_{a, \lambda, \ell} Z_{b, \ell, \eta}=\delta_{a, b} Z_{a, \lambda, \eta},
$$


in which summation over the direction index $\ell=1,2, \ldots, d$ is, of course, implied. Similarly the formulae

$$
\sum_{a=1}^{n} U_{a, \lambda, \eta}^{\dagger}=U_{\lambda, \eta}, \quad \sum_{a=1}^{n} V_{a, \lambda, \eta}^{\dagger}=V_{\lambda, \eta}
$$

follow from (2.9).

The geometric matrices and the supplementary matrices are, in fact, singular. From the relations (4.5) amongst the fields we have

$$
\begin{gathered}
\sum_{c=1}^{n} U_{\lambda, \eta}=\sum_{c=1}^{n} V_{\lambda, \eta}=\sum_{c=1}^{n} Z_{a, \lambda, \eta}=0 \\
\sum_{c=1}^{n} U_{a, \lambda, \eta}^{\dagger}=\sum_{c=1}^{n} V_{a, \lambda, \eta}^{\dagger}=\sum_{c=1}^{n} Z_{1, a \lambda, b \eta}^{\dagger}=\sum_{c=1}^{n} Z_{2, a \lambda, b \eta}^{\dagger}=0
\end{gathered}
$$

for any component index $c \in \lambda$ or $c \in \eta$. This suggests we choose some component, say our reference medium $q$, and let

$$
\begin{gathered}
U \equiv U_{\lambda, \eta}^{(q)}, \quad V \equiv V_{\lambda, \eta}^{(q)}, \quad Z_{a} \equiv Z_{a, \lambda, \eta}^{(q)}, \\
U_{a}^{\dagger} \equiv U_{a, \lambda, \eta}^{\dagger(q)}, \quad V_{a}^{\dagger} \equiv V_{a, \lambda, \eta}^{\dagger(q)}, \quad Z_{1, a, b}^{\dagger} \equiv Z_{1, a \lambda, b \eta}^{\dagger(q)}, \quad Z_{2, a, b}^{\dagger} \equiv Z_{2, a \lambda, b \eta}^{\dagger(q)},
\end{gathered}
$$

denote truncations of the full geometric matrices, defined with respect to the basis fields $\mathbf{E}_{\tau}^{(q)}, \mathbf{J}_{\tau}^{(q)}$, and $\mathbf{P}_{a \tau}^{(q)}$ : each component index, $c \in \lambda$ or $c \in \eta$ in these matrices runs over the set $\{1,2, \ldots, q-1, q+1, \ldots, n\}$, skipping the value $q$, while the indices $a$ and $b$ can equal $q$.

We will assume, unless otherwise explicitly stated, that the truncated basic geometric matrices $U, V$, and $Z_{a}, a=1,2, \ldots, n$, are non-singular with inverses $\tilde{U}, \tilde{V}$, and $\tilde{Z}_{a}$ satisfying

$$
\tilde{U}_{\lambda, \tau}^{(q)} U_{\tau, \eta}^{(q)}=\tilde{V}_{\lambda, \tau}^{(q)} V_{\tau, \eta}^{(q)}=\tilde{Z}_{a, \lambda, \tau}^{(q)} Z_{a, \tau, \eta}^{(q)}=\delta_{\lambda \eta}
$$

in which summation over $a$ is not implied, by our summation convention. This generic non-singularity condition is not serious and avoids exceptional cases that would prolong the discussion of the main point.

Note that the truncated geometric matrices contain the same information for any choice of reference medium $q$ as the full matrices: one can easily deduce the remaining elements in the full matrices from the identities (5.10) and (5.11).

\section{Expressions for the Supplementary Matrices}

Here we establish expressions for all the supplementary geometric matrices $U_{a}^{\dagger}, V_{a}^{\dagger}$, $Z_{1}^{\dagger}$, and $Z_{2}^{\dagger}$ in terms of the basic geometric matrices $U, V$, and $Z_{a}$. This shows that only the basic geometric matrices are needed to describe the relative orientation of the subspaces. The argument is simple and hinges on various relations between the hierarchies of fields $\mathbf{E}_{\tau}, \mathbf{J}_{\tau}$, and $\mathbf{P}_{a \tau}$.

By construction $\mathbf{P}_{a \tau}$ and $\mathbf{E}_{\tau}$ are the components of $\chi_{a} \mathbf{E}_{\tau}$ and $\Gamma_{1} \mathbf{P}_{\tau}$ that are orthogonal to the fields in $\mathscr{P}_{a, o(\tau)}^{<}$and $\mathscr{E}_{o(\tau)}^{<}$respectively. The appropriate linear combinations of fields necessary to ensure this orthogonality are, in fact, easy to 
find: given any $\tau$ with $o(\tau) \geqq 1$, let us establish the identities:

$$
\begin{aligned}
\mathbf{P}_{a \tau} & =\chi_{a} \mathbf{E}_{\tau}-\sum_{\mu, \sigma<\tau} \mathbf{P}_{a \sigma}^{(q)} \tilde{Z}_{a, \sigma, \mu}^{(q)}\left\langle\mathbf{P}_{a \mu} \mid \mathbf{E}_{\tau}\right\rangle, \\
\mathbf{E}_{\tau} & =\Gamma_{1} \mathbf{P}_{\tau}-\sum_{\mu, \sigma<\tau} \mathbf{E}_{\sigma}^{(q)} \tilde{U}_{\sigma, \mu}^{(q)}\left\langle\mathbf{E}_{\mu} \mid \mathbf{P}_{\tau}\right\rangle,
\end{aligned}
$$

where the inequality $\mu, \sigma<\tau$ implies the sum extends only over those sets of indices $\mu, \sigma$ with $o(\mu), o(\sigma)<o(\tau)$. To prove (6.1) it is only necessary to show that $\mathbf{P}_{a \tau}$ given by (6.1) is orthogonal to all the lower order fields in $\mathscr{P}_{a, o(a \tau)}^{<}$. By taking the inner product with any field $\mathbf{P}_{a \omega}^{(q)}$ such that $o(\omega)<o(\tau)$, we obtain the result

$$
\left\langle\mathbf{P}_{a \omega}^{(q)} \mid \mathbf{P}_{a \tau}\right\rangle=\left\langle\mathbf{P}_{a \omega}^{(q)} \mid \mathbf{E}_{\tau}\right\rangle-Z_{a, \omega, \sigma}^{(q)} \widetilde{Z}_{a, \sigma, \mu}^{(q)}\left\langle\mathbf{P}_{a \mu} \mid \mathbf{E}_{\tau}\right\rangle,
$$

which clearly vanishes and thus establishes (6.1). The relation (6.2) is proved by similar argument.

Now by taking the inner product of (6.1) with any field $\mathbf{P}_{a \omega}$ with $o(\omega) \geqq o(\tau)$, we deduce

$$
\begin{aligned}
\left\langle\mathbf{P}_{a \omega} \mid \mathbf{E}_{\tau}\right\rangle & =Z_{a, \omega, \tau} & & \text { when } & & o(\omega)=o(\tau) \\
& =0 & & \text { when } & & o(\omega)>o(\tau) .
\end{aligned}
$$

Similarly, the second identity implies

$$
\begin{aligned}
\left\langle\mathbf{P}_{\tau} \mid \mathbf{E}_{\omega}\right\rangle & =U_{\tau, \omega} & & \text { when } & & o(\omega)=o(\tau) \\
& =0 & & \text { when } & & o(\omega)>o(\tau) .
\end{aligned}
$$

We have thus obtained a complete expression for the inner product matrix $\left\langle\mathbf{P}_{\mu} \mid \mathbf{E}_{\sigma}\right\rangle$ in terms of the basic geometric matrices $U$ and $Z_{a}$. Now by taking the inner product of (6.1) with an electric field $\mathbf{E}_{\omega}$ rather than $\mathbf{P}_{a \omega}$ and by taking the inner product of (6.2) with $\mathbf{P}_{\omega}$ rather than $\mathbf{E}_{\omega}$, we obtain two more important relations, namely

$$
\begin{aligned}
& U_{a, \tau, \omega}^{\dagger}=\left\langle\mathbf{E}_{\omega} \mid \mathbf{P}_{a \tau}\right\rangle+\sum_{\mu, \sigma<\tau}\left\langle\mathbf{E}_{\omega} \mid \mathbf{P}_{a \sigma}\right\rangle \widetilde{Z}_{a, \sigma, \mu}^{(q)}\left\langle\mathbf{P}_{a \mu} \mid \mathbf{E}_{\tau}\right\rangle, \\
& Z_{1, \omega, \tau}^{\dagger}=\left\langle\mathbf{E}_{\tau} \mid \mathbf{P}_{\omega}\right\rangle+\sum_{\mu, \sigma<\tau}\left\langle\mathbf{P}_{\omega} \mid \mathbf{E}_{\sigma}\right\rangle \tilde{U}_{a, \sigma, \mu}^{(q)}\left\langle\mathbf{E}_{\mu} \mid \mathbf{P}_{\tau}\right\rangle .
\end{aligned}
$$

When combined with the above expression for $\left\langle\mathbf{P}_{\mu} \mid \mathbf{E}_{\sigma}\right\rangle$ the first relation gives

$$
U_{a, \tau, \omega}^{\dagger}=U_{a \tau, \omega}+U_{\tau, a \omega}+Z_{a, \tau, \omega}+U_{\tau, a \sigma} \tilde{Z}_{a, \sigma, \mu}^{(a)} U_{a \mu, \omega},
$$

or equivalently, in symbolic form,

$$
U_{a}^{\dagger}=U_{a}+U_{a}^{T}+Z_{a}+U_{a}^{T} Z_{a}^{-1} U_{a}
$$

where $U_{a}^{\dagger}$ and $Z_{a}$ are matrices that have elements $U_{a, \tau, \omega}^{\dagger(q)}$ and $Z_{a, \tau, \omega}^{(q)}$ labelled by $\tau$ and $\omega$, while for each $a=1,2, \ldots, n, U_{a}$, with transpose $U_{a}^{T}$, is the submatrix of $U$ that has elements $U_{a \tau, \omega}$, which are non-zero only when $o(\omega)=o(\tau)+1$. In this sense $U_{a}$ is block-superdiagonal matrix, $U_{a}^{T}$ being a block-subdiagonal matrix. Thus the matrix $U_{a}^{\dagger}$ is block-tridiagonal: its block structure is of the Jacobi form [1, 10].

Similarly, the second identity (6.7) implies

$$
Z_{1, a \tau, b \omega}^{\dagger}=Z_{b, a \tau, \omega}+Z_{a, \tau, b \omega}+U_{a \tau, b \omega}+Z_{a, \tau, \sigma} \tilde{U}_{\sigma, \mu}^{(q)} Z_{b, \mu, \omega},
$$


unless $o(\tau)=o(\omega)=0$, in which case

$$
Z_{1, a \ell, b \ell}^{\dagger}=U_{a \ell, b \ell} .
$$

Equation (6.10) can be written symbolically as

$$
Z_{1, a, b}^{\dagger}=Z_{b, a}+Z_{a, b}^{T}+U_{a, b}+Z_{a} U^{-1} Z_{b},
$$

in which $Z_{b, a}$ is, for each pair of component indices $a$ and $b$, a block-superdiagonal submatrix of $Z_{b}$, with elements $Z_{b, a \tau, \omega}$ labelled by $\tau$ and $\omega$.

By considering the fields $\mathbf{J}_{\tau}$ and $\mathbf{P}_{a \tau}$ we similarly deduce that

$$
\left\langle\mathbf{P}_{a \omega} \mid \mathbf{J}_{\tau}\right\rangle=V_{a \omega, \tau}-Z_{a, \omega, \tau},
$$

and thereby arrive at the block-tridiagonal expression

$$
V_{a}^{\dagger}=-V_{a}-V_{a}^{T}+Z_{a}+V_{a}^{T} Z_{a}^{-1} V_{a}
$$

for $V_{a}^{\dagger}$ in terms of $Z_{a}$ and $V$ and at the block-tridiagonal expression

$$
\begin{gathered}
Z_{2, a, b}^{\dagger}=-Z_{b, a}-Z_{a, b}^{T}+V_{a, b}+Z_{a} V^{-1} Z_{b}, \\
Z_{2, a \ell, b \hbar}^{\dagger}=V_{a \ell, b \hbar}
\end{gathered}
$$

for $Z_{2}^{\dagger}$ in terms of $Z_{a}$ and $V$. Thus we have obtained formulas for all the supplementary geometric parameters in terms of the basic geometric parameters.

An additional useful relation,

$$
\left\langle\mathbf{J}_{\omega}\left|\chi_{a}\right| \mathbf{E}_{\tau}\right\rangle=V_{\omega, a \tau}-Z_{a, \omega, \tau}-U_{a \omega, \tau}+V_{\omega, a \sigma} \tilde{Z}_{a, \sigma, \mu}^{(q)} U_{a \mu, \tau},
$$

is obtained by taking the inner product of (6.1) with the current field $\mathbf{J}_{\omega}$.

\section{Relations Between the Basic Matrices}

The basic geometric parameters themselves are not, in fact, independent. From (6.8) and (5.9) [and from (6.14) and (5.9)] we deduce

$$
\begin{aligned}
\sum_{a=1}^{n} Z_{a, \lambda, \eta} & =U_{\lambda, \eta}-\sum_{a=1}^{n} U_{\lambda, a \omega} \tilde{Z}_{a, \omega, \tau}^{(q)} U_{a \tau, \eta} \\
& =V_{\lambda, \eta}-\sum_{a=1}^{n} V_{\lambda, a \omega} \widetilde{Z}_{a, \omega, \tau}^{(q)} V_{a \tau, \eta}
\end{aligned}
$$

and (5.8) with (6.12) and (6.15) implies

$$
U_{a, b}+V_{a, b}=\delta_{a b} Z_{a}-Z_{a}(\tilde{U}+\tilde{V}) Z_{b}
$$

while (5.8) with (6.11) and (6.16) gives the result

$$
U_{a \ell, b \ell}+V_{a \ell, b \ell}=\delta_{a b} Z_{a, \ell, \ell}-Z_{a, \ell, m} Z_{b, m, \ell} .
$$

These relations are somewhat complicated and it is not obvious how we can generate sets of positive definite basic geometric matrices compatible with them. Our aim here is to reexpress the relations in a simpler form and to develop a scheme for generating all such sets of allowable basic geometric matrices. 
From (7.1) and (5.10), $U$ and $V$ are solutions for $X$ of the quadratic matrix equation

in which

$$
\begin{gathered}
X H X-X+G=0 \\
G \equiv \sum_{a=1}^{n} Z_{a}, \quad H_{a, b} \equiv Z_{q}^{-1}+\delta_{a b} Z_{a}^{-1} .
\end{gathered}
$$

By completing the square we obtain

$$
\left[\hat{H}\left(X-\frac{1}{2} \tilde{H}\right) \hat{H}\right]^{2}=\frac{1}{4} l-\hat{H} G \hat{A},
$$

where $\tilde{H}$ and $\hat{H}$ are the inverse and square root of $H$. This has multiple solutions for $X$, namely

$$
X=\frac{1}{2} \tilde{H} \pm \dot{H} \Psi\left[\frac{1}{4} I-\hat{A} G \hat{A}\right]^{1 / 2} \dot{H},
$$

in which $\dot{H}$ is the inverse of $A$ and $\psi$ is any symmetric, block-diagonal matrix commuting with $\hat{A G A}$, such that

$$
\Psi^{2}=I
$$

Hence $\psi$ must share a common set of eigenvectors with $\hat{A} G \hat{A}$, with eigenvalues \pm 1 .

The matrices $U$ and $V$ in fact correspond to opposite roots of Eq. (7.4), unless the roots of the equation happen to coincide. That is, for an appropriate choice of $\Psi$, we can identify the + sign solution in (7.7) with $U$ and the - sign solution with $V$. This result is a consequence of the relations (7.2) and (7.3) satisfied by $U$ and $V$ and is established by induction. Let $U^{(j)}, V^{(j)}, Z_{a}^{(j)}, G^{(j)}$, and $H^{(j)}$ denote those submatrices along the block diagonals of $U, V, \ldots$, and $H$, with elements $U_{\tau, \omega}^{(q)}, V_{\tau, \omega}^{(q)}$, $Z_{a, \tau, \omega}^{(q)}, G_{\tau, \omega}^{(q)}$, and $H_{\tau, \omega}^{(q)}$ satisfying $o(\tau)=o(\omega)=j$. To show $U$ and $V$ correspond to opposite roots of (7.4) we need to prove that for all $j$,

$$
U^{(j)}+V^{(j)}=\tilde{H}^{(j)}
$$

First we establish that any symmetric matrix $D$ of the form

has inverse $\tilde{D}$ given by

$$
D_{a, b}=\delta_{a b} A_{a}+B_{a}^{T} C^{-1} B_{b}
$$

$$
\widetilde{D}_{a, b}=\delta_{a b} A_{a}^{-1}-A_{a}^{-1} B_{a}^{T}\left(C+\sum_{g \neq q} B_{g} A_{g}^{-1} B_{g}^{T}\right)^{-1} B_{b} A_{b}^{-1} .
$$

This is accomplished by taking the product of $D_{a, b}$ with $\widetilde{D}_{b, c}$, given by (7.11), and summing over $b(\neq q)$ to obtain the expression

$$
\delta_{a c} I=\delta_{a c} I+B_{a}^{T} C^{-1}\left[I-\left(C+\sum_{b \neq q} B_{b} A_{b}^{-1} B_{b}^{T}\right)\left(C+\sum_{g \neq q} B_{g} A_{g}^{-1} B_{g}^{T}\right)^{-1}\right] B_{c} A_{c}^{-1},
$$

which is clearly an identity. Now $H$, defined by (7.5), is of this form (7.10) with $A_{a}=Z_{a}^{-1}, C=Z_{q}$, and $B_{a}=I$ (for all $a$ ) and hence its inverse is

$$
\tilde{H}_{a, b}=\delta_{a b} Z_{a}-Z_{a} \widetilde{G} Z_{b} \text {. }
$$

It thus remains to prove

$$
U_{a, b}^{(j)}+V_{a, b}^{(j)}=\delta_{a b} Z_{a}^{(j-1)}-Z_{a}^{(j-1)} \widetilde{G}^{(j-1)} Z_{b}^{(j-1)},
$$


for all $j$. Clearly (7.3), (5.4), and (7.5) imply this relation is satisfied when $j=1$. So suppose (7.14) and hence (7.9), holds when $j=g$ for some $g$. By substituting (7.9) in (7.4) and taking $X=U$ we have

$$
G^{(g)}=U^{(g)}-U^{(g)}\left(U^{(g)}+V^{(g)}\right)^{-1} U^{(g)},
$$

which implies

$$
\widetilde{G}^{(g)}=\widetilde{U}^{(g)}+\widetilde{\nabla}^{(g)} .
$$

By inserting this back in (7.2) we establish that our proposed relation (7.14) holds when $j=g+1$. By induction on $j,(7.9)$ and (7.16) are satisfied for all integers $j$ and g. Conversely, the initial equations (7.1)-(7.3) must hold whenever $U, V$, and $Z_{a}$ are chosen compatible with (7.9) and (7.16).

From the definitions (5.1) it is clear that the basic geometric parameters are positive semidefinite matrices. Any set of (block-diagonal, symmetric, real) basic geometric matrices that satisfy the positivity conditions,

$$
U \geqq 0, \quad V \geqq 0, \quad Z_{a} \geqq 0 \quad \forall a,
$$

the consistency relations (7.9) and (7.16) [which imply (7.1)-(7.3)] and the additional orthogonality constraint that

$$
\sum_{a=1}^{n} Z_{a, \ell, k}=\delta_{\ell k}
$$

will be called allowable. Clearly any actual composite corresponds to an allowable set of basic geometric parameters. Thus the set of allowable geometric parameters encompasses the set of physically realizable geometric parameters. The converse, however, is not true. In particular, an allowable set of geometric parameters need not satisfy (5.4) or (5.6): clearly the orthogonality constraint (7.18) is a weaker condition that (5.4). Nevertheless, as we will see in Sect. 8, any set of allowable geometric parameters does describe the orientation in some Hilbert space $\mathscr{H}$ of three appropriately chosen orthogonal subspaces $\mathscr{U}, \mathscr{E}$, and $\mathscr{J}$, spanning $\mathscr{H}$, with respect to $n$ other mutually orthogonal subspaces $\mathscr{P}_{a}, a=1,2, \ldots, n$, spanning $\mathscr{H}$. Thus the "effective tensor" $\sigma\left(\sigma_{1}, \sigma_{2}, \ldots, \sigma_{n}\right)$ is still well-defined [via (2.11) and (1.1)] for any allowable set of geometric parameters, and for any set of variables $\sigma_{a}, a=1,2, \ldots, n$, located in an open half of the complex plane.

It is now apparent from (7.7) how we can generate all possible allowable sets of basic geometric parameters. We begin by choosing any set of $n$ positive semidefinite symmetric real $d \times d$ matrices $Z_{a}^{(0)}, a=1,2, \ldots, n$, satisfying (7.18). Next, supposing the matrices $Z_{a}^{(j-1)}$ have been specified, for some $j \geqq 1$, we choose any set of $n$ symmetric real $d(n-1)^{j}$-dimensional matrices $Z_{a}^{(j)}$ satisfying the inequalities

$$
\frac{1}{4} \tilde{H}^{(j)} \geqq \sum_{a=1}^{n} Z_{a}^{(j)}, \quad Z_{a}^{(j)} \geqq 0,
$$

where $\widetilde{H}^{(j)}$ is calculated from the matrices $Z_{a}^{(j-1)}$ via (7.5). This constraint is necessary to ensure (7.7) has real, positive definite solutions for $U^{(j)}$ and $V^{(j)}$ in terms of the matrix $\psi^{(j)}$, that is in turn selected to be any matrix sharing a common set of eigenvectors with $A^{(j)} G^{(j)} \hat{A}^{(j)}$ having eigenvalues \pm 1 . By repeating this procedure for successive values of $j$, starting from $j=1$, we obtain an allowable set 
of basic geometric parameters. Conversely, any allowable set of geometric parameters can clearly be constructed in this manner for an appropriate choice of the matrices $Z_{a}$ and $\Psi$.

In this scheme for generating allowable basic parameters we need to know the matrices $Z_{a}^{(j-1)}$ before selecting a possible set of matrices $Z_{a}^{(j)}$. Thus, the constraints on the geometric parameters are coupled between different levels in the hierarchy. In the next section we show how these constraints can be decoupled by choosing a different basis set of fields (or "vectors") in $\mathscr{H}$. The set of allowable basic geometric matrices are parametrized in terms of a block-diagonal, symmetric set of fundamental geometric matrices, comprised of normalization factors, $N=N_{\lambda, \eta}^{(q)}$ and weights $W_{a}=W_{a, \lambda, \eta}^{(q)}$, satisfying the relatively simple constraints

$$
N \geqq 0, \quad W_{a} \geqq 0, \quad \sum_{a=1}^{n} W_{a}=l .
$$

These normalization and weight matrices represent a minimal set of invariants that characterize the relative orientation of the subspaces. In Sect. 9 we establish that the eigenvalues of these matrices are preserved under a change of reference medium which demonstrates that an allowable set of geometric parameters remains allowable under a change of reference medium.

\section{The Fundamental Matrices and a Canonical Basis Set of Fields}

To isolate the essential geometric properties characterizing the relative orientation of the subspaces we need to simplify the form of the relations between the basic geometric parameters. This is accomplished by transforming the basis sets $\mathbf{E}_{\tau}^{(q)}, \mathbf{J}_{\tau}^{(q)}$, and $\mathbf{P}_{a \tau}^{(q)}$ into three new block-orthogonal families $\mathbf{E}_{\tau}^{\prime(q)}, \mathbf{J}_{\tau}^{\prime(q)}$, and $\mathbf{P}_{a \tau}^{\prime(q)}$, related to the old fields via the special linear transformations,

$$
\begin{array}{rlrl}
\mathbf{E}_{\tau}^{\prime(q)}=L_{\tau, \omega}^{(q)} \mathbf{E}_{\omega}^{(q)}, & \mathbf{E}_{\eta}^{(q)}=\widetilde{L}_{\eta, \tau}^{(q)} \mathbf{E}_{\tau}^{\prime(q)}, & \mathbf{J}_{\tau}^{\prime(q)}=L_{\tau, \omega}^{(q)} \mathbf{J}_{a \omega}^{(q)}, & \mathbf{J}_{\eta}^{(q)}=\widetilde{L}_{\eta, \tau}^{(q)} \mathbf{J}_{\tau}^{\prime(q)}, \\
\mathbf{P}_{a \tau}^{\prime(q)}=K_{\tau, \omega}^{(q)} \mathbf{P}_{a \omega}^{(q)}, & \mathbf{P}_{a \eta}^{(q)}=\widetilde{K}_{\eta, \tau}^{(q)} \mathbf{P}_{a \tau}^{\prime(q)},
\end{array}
$$

where the not necessarily symmetric transformation matrices $L \equiv(\tilde{L})^{-1}, K \equiv(\widetilde{K})^{-1}$ are selected to be block-diagonal to preserve the block orthogonality of the fields. Note that the electric and current fields are transformed by the same matrix $L$, while the matrix $K$ transforming the fields $\mathbf{P}_{a \tau}^{(q)}$ is independent of the component index $a$. Under this mapping the basic geometric parameters $U, V$, and $Z_{a}$ are transformed into a new set $U^{\prime}, V^{\prime}$, and $Z_{a}^{\prime}$. We seek a set of transformation matrices $L$ and $K$ such that the new basic geometric parameters have the summation properties

$$
U^{\prime}+V^{\prime}=I, \quad \sum_{a=1}^{n} Z_{a}^{\prime}=I
$$

which is a natural requirement considering that the relations (7.9) and (7.16) that we wish to simplify incorporate the matrix combinations $U+V$ and $\sum_{a=1}^{n} Z_{a}$. Any such set of matrices satisfying (8.2) can of course be replaced by a set of normalization factors, $N_{\lambda, \eta}^{(q)}$, and a set of weights, $W_{a, \lambda, \eta}^{(q)}$, defined implicitly through 
the equations

$$
\begin{aligned}
& U^{\prime}=(I+N)^{-1}, \\
& V^{\prime}=\left(I+N^{-1}\right)^{-1}, \\
& Z_{a}^{\prime}=W_{a},
\end{aligned}
$$

where

$$
\sum_{a=1}^{n} W_{a}=l
$$

The motivation for introducing these normalization factors and weights is simply that they appear to play a natural role in continued fraction expansions of the effective conductivity: they are the generalizations, to anisotropic multicomponent composites, of the weights and normalization constants introduced for two-phase isotropic composites [12].

One significant consequence of the requirement (8.2) is that it ensures the fields

$$
\mathbf{x}_{\omega}^{(q)} \equiv \sum_{a=1}^{n} \mathbf{P}_{a \omega}^{\prime(q)}, \quad \mathbf{y}_{\tau}^{(q)} \equiv \mathbf{E}_{\tau}^{\prime(q)}+\mathbf{J}_{\tau}^{(q)}
$$

when taken together and defined for all strings $\omega$ and $\tau$ with $o(\omega) \geqq 0$ and $o(\tau) \geqq 1$ form an orthonormal basis set for $\mathscr{H}$, which we denote as a canonical basis set. (Other canonical basis sets are obtained by selecting a different reference medium or by choosing a different basis set of uniform fields $\mathbf{x}_{\ell}, \ell=1,2, \ldots, d$.) To prove this first note that the orthonormality

$$
\left\langle\mathbf{x}_{\omega}^{(q)} \mid \mathbf{x}_{\eta}^{(q)}\right\rangle=\delta_{\omega \eta}, \quad\left\langle\mathbf{y}_{\tau}^{(q)} \mid \mathbf{y}_{\lambda}^{(q)}\right\rangle=\delta_{\tau \lambda}, \quad\left\langle\mathbf{x}_{\omega}^{(q)} \mid \mathbf{y}_{\tau}^{(q)}\right\rangle=0
$$

of these fields is established by substituting (8.7) in (8.8) and using (8.2), (8.1), and (5.10) together with the expressions (6.4), (6.5), and (6.13) for the inner products $\left\langle\mathbf{P}_{a \omega}^{(q)} \mid \mathbf{E}_{\tau}^{(q)}\right\rangle$ and $\left\langle\mathbf{P}_{a \omega}^{(q)} \mid \mathbf{J}_{\tau}^{(q)}\right\rangle$. Next note that the $d$ independent uniform fields $\mathbf{x}_{\ell}^{(q)}=K_{\ell k}^{(q)} \mathbf{x}_{k}, \ell=1,2, \ldots, d$, clearly span $\mathscr{U}$. Subsequently, a simple counting argument establishes that for any given integer $g \geqq 1$ the $2 k \equiv 2 d(n-1)^{g}$ independent fields $\mathbf{x}_{\tau}^{(q)}$ and $\mathbf{y}_{\lambda}^{(q)}$ with $o(\tau)=o(\lambda)=g$ span the same subspace of $\mathscr{E} \oplus \mathscr{J}$ that is spanned by the $2 k$ independent fields $\mathbf{E}_{\sigma}^{(q)}$ and $\mathbf{J}_{\mu}^{(q)}$ with $o(\sigma)=o(\mu)=g$. Therefore the fields $\mathbf{x}_{\omega}^{(q)}$ and $\mathbf{y}_{\tau}^{(q)}$ with $o(\omega) \geqq 0$ and $o(\tau) \geqq 1$ span $\mathscr{H}$.

It is not a priori clear from the definitions (8.7) why the fields $\mathbf{x}_{\omega}^{(q)}$ and $\mathbf{y}_{\tau}^{(q)}$ should form an orthonormal basis set. In fact there exists a much simpler way of defining these fields and the weights and normalization matrices that avoids much of the lengthy analysis of Sects. 3-8: this alternative formulation is discussed in Appendix 1.

Of course, we still need to establish that suitable transformation matrices $L$ and $K$ exist. From (7.9), (7.16) and the defining equations (7.5) for $G$ and $H$ we have

where

$$
\begin{gathered}
G^{-1}=K^{T} K=L^{T}\left[2 I+N+N^{-1}\right] L, \\
H_{a, b}=\sum_{c \neq q} L_{a, c}^{T} L_{c, b}=K^{T} \tilde{Y}_{a, b} K,
\end{gathered}
$$

$$
\tilde{Y}_{a, b} \equiv W_{q}^{-1}+\delta_{a b} W_{a}^{-1}
$$


and $H_{a, b}$ and $L_{a, b}$, for each pair of component indices $a$ and $b$, are those submatrices of $H$ and $L$ with elements $H_{a \lambda, b \eta}^{(q)}$ and $L_{a \lambda, b \eta}^{(q)}$. To ensure the relations (8.9)-(8.11) are satisfied we make the obvious choice and select $K$ and $L$ such that

$$
\begin{gathered}
K=(\hat{N}+\dot{N}) L ; \quad \hat{N} \equiv(\dot{N})^{-1} \equiv N^{1 / 2} \\
L_{a, b}=\dot{Y}_{a, b} K
\end{gathered}
$$

where $\dot{Y}=\dot{Y}_{\omega, \tau}^{(q)}$ denotes the positive definite symmetric matrix that is the square root of $\tilde{Y}$, satisfying

$$
\sum_{b \neq q} \dot{Y}_{a, b} \dot{Y}_{b, c}=\tilde{Y}_{a, c}
$$

These equations, together with the identities

$$
\begin{gathered}
N=\left(L U L^{T}\right)^{-1}-I, \\
W_{a}=K Z_{a} K^{T},
\end{gathered}
$$

implied by (8.1), (8.3), and (8.5), serve to define by recursion the transformation matrices $K$ and $L$, the normalization factors $N$, and the weights $W_{a}$. To see this, let $\dot{Y}^{(j)}, K^{(j)}, L^{(j)}, N^{(j)}$, and $W_{a}^{(j)}$ denote those submatrices along the block diagonals of $\dot{Y}, K, L, N$, and $W_{a}$ that have elements $\dot{Y}_{\tau, \omega}^{(q)}, K_{\tau, \omega}^{(q)}, L_{\tau, \omega}^{(q)}, N_{\tau, \omega}^{(q)}, W_{a, \tau, \omega}^{(q)}$ with $o(\tau)=o(\omega)=j$. Now if $K^{(j)}$ is known for some integer $j$ then (8.16) enables $W_{a}^{(j)}$ and hence $\dot{Y}^{(j+1)}$ to be calculated, via (8.11). Then from $(8.13) L^{(j+1)}$ can be determined, which with $(8.15)$ gives $N^{(j+1)}$. Subsequently through $(8.12)$ we deduce $K^{(j+1)}$.

To complete this definition the initial matrix $K^{(0)}$ needs to be specified: for simplicity, the natural choice

$$
K_{\ell, k}^{(0)} \equiv \delta_{\ell k}
$$

is made, which with (8.7) implies that $\mathbf{x}_{\ell}^{(q)}=\mathbf{x}_{\ell}$, for $\ell=1,2, \ldots, d$. This gives a set of elementary weights

$$
W_{a, \ell, k}^{(q)}=Z_{a, \ell, k}^{(q)}
$$

satisfying (8.6) as a consequence of (7.18).

Conversely, by similar inductive reasoning, if $N$ and $W_{a}$ are known, then the initial equation (8.17) and the relations (8.12), (8.13) with the identities

$$
\begin{aligned}
U & =\tilde{L}(I+N)^{-1} \tilde{L}^{T}, \\
V & =\tilde{L}\left(I+N^{-1}\right)^{-1} \tilde{L}^{T}, \\
Z_{a} & =\widetilde{K} W_{a} \widetilde{K}^{T},
\end{aligned}
$$

which follow from (8.3)-(8.5), enable the basic geometric parameters $U, V$, and $Z_{a}$ and the transformation matrices $K$ and $L$ to be recovered. Moreover, any blockdiagonal choice of $N$ and $W_{a}$ satisfying (8.6) will yield a set of basic geometric parameters consistent with (7.1)-(7.3): this can be verified by direct substitution, and has been ensured by the choice of $K$ and $L$ compatible with (8.9) and (8.10).

Now the positivity of the matrix $U$ implies the eigenvalues of $N$ lie in the interval $(-1, \infty)$, while the positivity of $V$ implies the eigenvalues of $N^{-1}$ are similarly confined to this interval. Therefore $N$ must be a positive-semidefinite 
matrix. Also, the positivity of the matrices $Z_{a}$ directly implies, via (8.5), the positivity of the weights $W_{a}$. Conversely, any choice of positive-semidefinite $N$ and $W_{a}$, leads via (8.19)-(8.21) to a positive-semidefinite set of geometric parameters, $U$, $V$, and $Z_{a}$.

Thus we have obtained a complete characterization of the set of allowable geometric matrices: they are the set of basic geometric matrices which follow from any choice of symmetric, positive-semidefinite, block-diagonal matrices $N$ and $W_{a}$ satisfying (8.6). For this reason the normalization factors and weights are called fundamental geometric parameters.

From (5.4) and (8.18) the elementary weights for a real composite material take the values

$$
W_{a, \ell, k}^{(q)}=f_{a} \delta_{\ell k},
$$

and (5.6) in conjunction with (8.15), (8.13), and (8.17) implies that the elementary matrices $U^{\prime(1)}$ and $V^{\prime(1)}$ satisfy

$$
U_{a \ell, b \ell}^{\prime(q)}=(d-1)^{-1} V_{a \ell, b \ell}^{\prime(q)}=\delta_{a b} .
$$

Consequently when the composite is isotropic the elementary normalization factor is given by

$$
N_{a \ell, b \ell}^{(q)}=(d-1) \delta_{a b} \delta_{\ell k},
$$

while for an isotropic two-component composite (8.23) implies that the eigenvalues $n_{\ell}^{(1)}, \ell=1,2, \ldots, d$, of the elementary normalization factor $N^{(1)}$ are related via the identity

$$
\sum_{\ell=1}^{d} 1 /\left(n_{\ell}^{(1)}+1\right)=1
$$

The projection operators $\Gamma_{i}, i=0,1,2$, and $\chi_{a}, a=1,2, \ldots, n$, have an elegant matrix representation in the canonical basis set (8.7) in terms of the normalization factors and weights. Let us introduce the geometric matrix

$$
Y_{a \lambda, b \eta}^{(q)} \equiv \delta_{a b} W_{a, \lambda, \eta}^{(q)}-W_{a, \lambda, \sigma}^{(q)} W_{b, \sigma, \eta}^{(q)},
$$

with positive square root $\hat{\gamma}$ defined by

$$
\sum_{b \neq q} \hat{Y}_{a \omega, b \tau}^{(q)} \hat{Y}_{b \tau, c \lambda}^{(q)}=Y_{a \omega, c \lambda}^{(q)} .
$$

These matrices $\hat{Y}$ and $Y$ are in fact the inverses of $\dot{Y}$ and $\tilde{Y}$, defined by (8.11) and (8.14): this clearly follows from a relation analogous to (7.13). Consequently from (8.12) and (8.13) we have

$$
\begin{gathered}
L \widetilde{K}=(\tilde{N}+\dot{N})^{-1}=\hat{O}^{\prime} \hat{V}^{\prime}, \\
K \tilde{L}_{a, b}=\hat{Y}_{a, b} .
\end{gathered}
$$

Now by using (8.7) and (8.1) to express the fields $\mathbf{x}_{\tau}^{(q)}$ in terms of the fields $\mathbf{P}_{a \omega}^{(q)}$ we deduce that for all strings $\tau$ and $\lambda$ with $o(\tau), o(\lambda)>1$,

$$
\left\langle\mathbf{x}_{\tau}^{(q)}\left|\Gamma_{1}\right| \mathbf{x}_{\lambda}^{(q)}\right\rangle=\sum_{a=1}^{n} \sum_{b=1}^{n} K_{\tau, \omega}^{(q)} Z_{1, a \omega, b \eta}^{\dagger(q)} K_{\eta, \lambda}^{(q)}=K_{\tau, \omega}^{(q)} G_{\omega, \sigma}^{(q)} \tilde{U}_{\sigma, \mu}^{(q)} G_{\mu, \eta}^{(q)} K_{\eta, \lambda}^{(q)},
$$


where the latter identity follows from (6.10), (5.10) and the definition (7.5) of $G$. In conjunction with (8.9) and (8.28) this implies

$$
\left\langle\mathbf{x}_{\tau}^{(q)}\left|\Gamma_{1}\right| \mathbf{x}_{\lambda}^{(q)}\right\rangle=\left\langle\mathbf{y}_{\tau}^{(q)}\left|\Gamma_{2}\right| \mathbf{y}_{\lambda}^{(q)}\right\rangle=V_{\tau, \lambda}^{\prime(q)} .
$$

The same approach gives the expressions

$$
\begin{aligned}
& \left\langle\mathbf{y}_{\tau}^{(q)}\left|\Gamma_{1}\right| \mathbf{y}_{\lambda}^{(q)}\right\rangle=\left\langle\mathbf{x}_{\tau}^{(q)}\left|\Gamma_{2}\right| \mathbf{x}_{\tau}^{(q)}\right\rangle=\mathbf{U}_{\tau, \lambda}^{(q)}, \\
& \left\langle\mathbf{x}_{\tau}^{(q)}\left|\Gamma_{1}\right| \mathbf{y}_{\lambda}^{(q)}\right\rangle=-\left\langle\mathbf{x}_{\tau}^{(q)}\left|\Gamma_{2}\right| \mathbf{y}_{\lambda}^{(q)}\right\rangle=\widehat{U}_{\tau, \sigma}^{(q)} \hat{V}_{\sigma, \lambda}^{(q)}, \\
& \left\langle\mathbf{x}_{\ell}^{(q)}\left|\Gamma_{1}\right| \mathbf{x}_{\ell}^{(q)}\right\rangle=\left\langle\mathbf{x}_{\ell}^{(q)}\left|\Gamma_{2}\right| \mathbf{x}_{k}^{(q)}\right\rangle=0,
\end{aligned}
$$

for the remaining matrix elements of the operators $\Gamma_{1}$ and $\Gamma_{2}$, and in conjunction with (6.8), (6.14), and (6.17) gives the expressions

$$
\begin{aligned}
\left\langle\mathbf{x}_{\omega}^{(q)}\left|\chi_{a}\right| \mathbf{x}_{\eta}^{(q)}\right\rangle & =W_{a \omega, \eta}^{(q)}, \\
\left\langle\mathbf{y}_{\omega}^{(q)}\left|\chi_{a}\right| \mathbf{y}_{\eta}^{(q)}\right\rangle & =\hat{Y}_{\tau, a \sigma} \tilde{W}_{a \mu, \lambda}^{(q)} \hat{Y}_{a \mu, \lambda}, \\
\left\langle\mathbf{x}_{\omega}^{(q)}\left|\chi_{a}\right| \mathbf{y}_{\tau}^{(q)}\right\rangle & =\hat{Y}_{a \omega, \tau},
\end{aligned}
$$

for the matrix elements of the operators $\chi_{a}$ : these formulae even apply when $a=q$, provided that we define

$$
\hat{Y}_{q \omega, \tau} \equiv-\sum_{b \neq q} \hat{Y}_{b \omega, \tau}^{(q)} .
$$

Since the elementary fields $\mathbf{x}_{\ell}^{(q)}=\mathbf{x}_{\ell}$ span $\mathscr{U}$, the matrix representing $\Gamma_{0}$ is especially simple. The only non-zero elements are

$$
\left\langle\mathbf{x}_{\ell}^{(q)}\left|\Gamma_{0}\right| \mathbf{x}_{k}^{(q)}\right\rangle=\delta_{\ell k} .
$$

In summary these matrix representations of the operators $\Gamma_{i}$ and $\chi_{a}$ have the block structure illustrated in Figs. 1 and 2. They can be expressed in terms of the

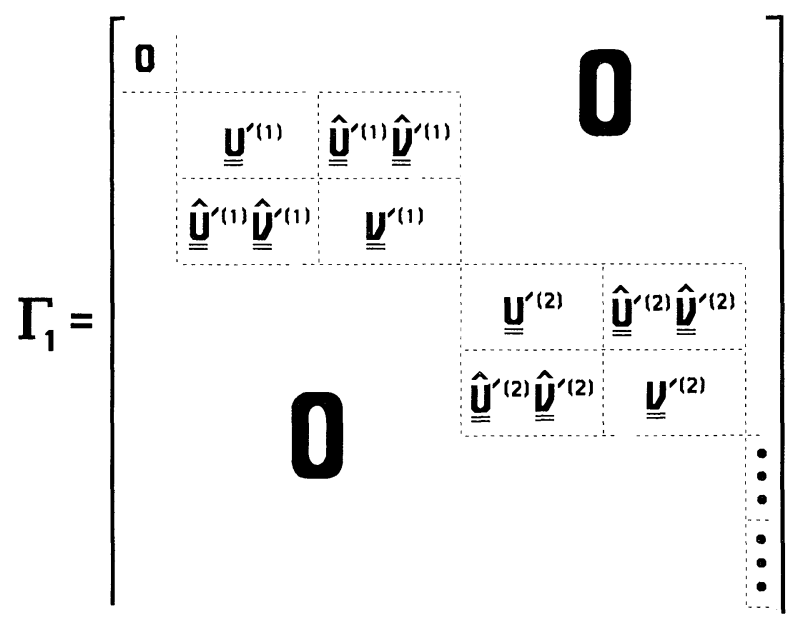

Fig. 1. Block structure of the matrix representing the projection operator $\Gamma_{1}$ in the canonical basis set (8.7). The matrix representing $\Gamma_{2}$ can be obtained from this matrix by interchanging $U^{(j)}$ and $V^{\prime(j)}$ and switching the signs of the off-diagonal matrix elements $\widehat{O}^{\prime(j)} \hat{V}^{\prime(j)}$ 


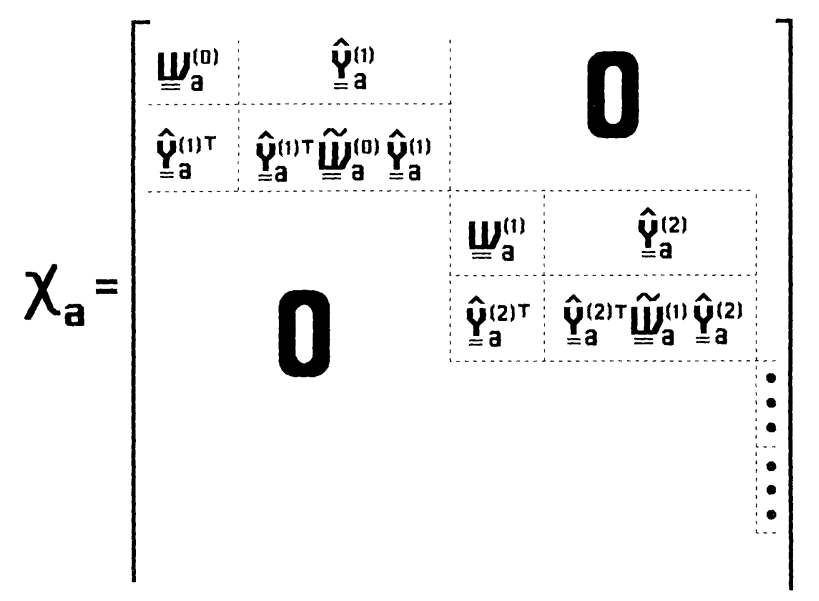

Fig. 2. Block structure of the matrices representing the projection operators $\chi_{a}$ in the canonical basis set (8.7)

normalization factors and weights by substituting (8.3), (8.4), and (8.11) in the above formulae. Furthermore it can be checked, by direct matrix multiplication, that the matrices represent projection operators satisfying (2.5), (2.6), (2.8), and (2.9) for any choice of allowable normalization factors and weights.

Thus when the geometric parameters are allowable (but not necessarily realizable by a real composite material) we can consider $\mathscr{H}$ as an infinite dimensional vector space and define $\mathscr{U}, \mathscr{E}, \mathscr{J}$, and $\mathscr{P}_{a}$, for $a=1,2, \ldots, n$, as those subspaces onto which the matrices representing $\Gamma_{0}, \Gamma_{1}, \Gamma_{2}$, and $\chi_{a}$ project. The field equation (2.11) and the effective tensor $\sigma^{*}$ are perfectly well-defined in this Hilbert space. Note that the matrix elements of $\Gamma_{1}$ and $\Gamma_{2}$ only depend on the normalization factors while the matrix elements of $\chi_{a}$ only depend on the weights.

Representations for the fields $\mathbf{E}_{\tau}^{(q)}$ and $\mathbf{J}_{\tau}^{\prime(q)}$ in this canonical basis set are obtained by projecting $\mathbf{y}_{\tau}^{(q)}$, defined by (8.7), onto the subspaces $\mathscr{E}$ and $\mathscr{J}$. From (8.31) and (8.32) this gives

$$
\begin{aligned}
& \mathbf{E}_{\tau}^{\prime(q)}=U_{\tau \lambda}^{\prime(q)} \mathbf{y}_{\lambda}^{(q)}+\hat{U}_{\tau \sigma}^{\prime(q)} \hat{V}_{\sigma \lambda}^{\prime(q)} \mathbf{x}_{\lambda}^{(q)}, \\
& \mathbf{J}_{\tau}^{\prime(q)}=V_{\tau \lambda}^{\prime(q)} \mathbf{y}_{\lambda}^{(q)}-\hat{U}_{\tau, \sigma}^{\prime(q)} \hat{V}_{\sigma \lambda}^{\prime(q)} \mathbf{x}_{\lambda}^{(q)} .
\end{aligned}
$$

Rather than working with these fields which have an awkward representation let us define

$$
\begin{aligned}
\mathbf{E}_{\eta}^{\prime \prime(q)} & =\tilde{U}_{\eta \lambda}^{\prime(q)} \mathbf{E}_{\tau}^{\prime(q)}=\mathbf{y}_{\eta}^{(q)}+\hat{N}_{\eta, \sigma}^{(q)} \mathbf{x}_{\sigma}^{(q)}, \\
\mathbf{J}_{\eta}^{\prime \prime(q)} & =\tilde{V}_{\eta, \lambda}^{\prime(q)} \mathbf{J}_{\lambda}^{\prime(q)}=\mathbf{y}_{\eta}^{(q)}-\dot{N}_{\eta, \sigma}^{(q)} \mathbf{x}_{\sigma}^{(q)}
\end{aligned}
$$

as two new sets of basis fields for $\mathscr{E}$ and $\mathscr{J}$, that have the advantage of a concise representation in the canonical basis set. Similarly we can define the polarization fields

$$
\mathbf{P}_{a \omega}^{\prime \prime(q)}=\tilde{W}_{a, \omega, \tau}^{(q)} \mathbf{P}_{a, \tau}^{\prime(q)}=\mathbf{x}_{\omega}^{(q)}+\tilde{W}_{a, \omega, \tau}^{(q)} \hat{Y}_{a \tau, \mu}^{(q)} \mathbf{y}_{\mu}^{(q)},
$$

with $o(\omega) \geqq 0$, as a new basis set for $\mathscr{P}_{a}$. 


\section{Effect of Changing the Reference Medium}

The fundamental geometric parameters, $W_{a} \equiv W_{a, \lambda, \eta}^{(q)}$ and $N \equiv N_{\lambda, \eta}^{(q)}$, were defined with respect to a specific reference medium labelled by the index $q$. Here we consider the type of transformations these parameters and matrices undergo as the reference medium is changed from component $q$ to say component $p \neq q$.

When the reference medium is changed the vector basis sets $\mathbf{E}_{\tau}^{(q)}, \mathbf{J}_{\tau}^{(q)}$, and $\mathbf{P}_{a \tau}^{(q)}$ are replaced by new vector basis sets

$$
\mathbf{E}_{\tau}^{(p)}=M_{\tau, \omega}^{(p, q)} \mathbf{E}_{\omega}^{(q)}, \quad \mathbf{J}_{\tau}^{(p)}=M_{\tau, \omega}^{(p, q)} \mathbf{J}_{\omega}^{(q)}, \quad \mathbf{P}_{a \tau}^{(p)}=M_{\tau, \omega}^{(p, q)} \mathbf{P}_{a \omega}^{(q)},
$$

where the block-diagonal transformation matrix $M$ is given from (4.5) through the recursion relations

$$
\begin{aligned}
M_{\ell, k}^{(p, q)} \equiv \delta_{\ell k}, & M_{a, b}^{(p, q)} \equiv \delta_{a b}-\delta_{a q}, \\
M_{a \tau, b \omega}^{(p, q)} \equiv M_{a, b}^{(p, q)} M_{\tau, \omega}^{(p, q)}, & M_{\tau, \omega}^{(p, q)} \equiv 0 \quad \text { for } \quad o(\tau) \neq o(\omega),
\end{aligned}
$$

which apply when $p \neq q$. In conjunction with (8.1) this implies

where

$$
\begin{gathered}
\mathbf{E}_{\tau}^{\prime(p)}=L_{\tau, \omega}^{\dagger(p, q)} \mathbf{E}_{\omega}^{\prime(q)}, \quad \mathbf{J}_{\tau}^{\prime(p)}=L_{\tau, \omega}^{\dagger(p, q)} \mathbf{J}_{\omega}^{\prime(q)}, \\
\mathbf{P}_{a \tau}^{\prime(p)}=K_{\tau, \omega}^{\dagger(p, q)} \mathbf{P}_{a \omega}^{\prime(q)},
\end{gathered}
$$

$$
\begin{gathered}
L_{\tau, \omega}^{\dagger(p, q)} \equiv L_{\tau, \eta}^{(p)} M_{\eta, \lambda}^{(p, q)} \widetilde{L}_{\lambda, \omega}^{(q)}, \\
K_{\tau, \omega}^{\dagger(p, q)} \equiv K_{\tau, \eta}^{(p)} M_{\eta, \lambda}^{(p, q)} \widetilde{K}_{\lambda, \omega}^{(q)} \equiv \delta_{\tau \omega} \quad \text { if } \quad o(\tau)=o(\omega)=0
\end{gathered}
$$

are block-diagonal linear transformations. From these relations we deduce

$$
\begin{gathered}
U_{\tau, \omega}^{\prime(p)}=L_{\tau, \eta}^{\dagger(p, q)} U_{\eta, \lambda}^{\prime(q)} L_{\lambda, \omega}^{\dagger(p, q) T}, \quad V_{\tau, \omega}^{\prime(p)}=L_{\tau, \eta}^{\dagger(p, q)} V_{\eta, \lambda}^{\prime(q)} L_{\lambda, \omega}^{\dagger(p, q) T}, \\
Z_{a, \tau, \omega}^{\prime(p)}=K_{\tau, \eta}^{\dagger(p, q)} Z_{a, \eta, \lambda}^{\prime(q)} K_{\lambda, \omega}^{\dagger(p, q) T},
\end{gathered}
$$

and by substitution in (8.2), the result

$$
L_{\tau, \eta}^{\dagger(p, q)} L_{\eta, \omega}^{\dagger(p, q) T}=\delta_{\tau \omega}, \quad K_{\tau, \eta}^{\dagger(p, q)} K_{\eta, \omega}^{\dagger(p, q) T}=\delta_{\tau \omega}
$$

follows. Thus $L^{\dagger}$ and $K^{\dagger}$ are block-diagonal orthogonal matrices. Consequently, from (9.5) and the definitions (8.3)-(8.5), the weights and normalization factors must transform linearly, satisfying

$$
N_{\tau, \omega}^{(p)}=L_{\tau, \eta}^{\dagger(p, q)} N_{\eta, \lambda}^{(q)} L_{\lambda, \omega}^{\dagger(p, q) T}, \quad W_{a, \tau, \omega}^{(p)}=K_{\tau, \eta}^{\dagger(p, q)} W_{a, \eta, \lambda}^{(q)} K_{\lambda, \omega}^{\dagger(p, q) T}
$$

Furthermore, since $L^{\dagger}$ and $K^{\dagger}$ are orthogonal, the eigenvalues of $N^{(j)}$ and $W_{a}^{(j)}$ must be preserved under changes of reference medium.

Now note that (8.28) and the transformation rules (9.7) for the normalization factors imply

$$
K_{\tau, \lambda}^{(p)} \widetilde{L}_{\lambda, \omega}^{(p)}=L_{\tau, \sigma}^{\dagger(p, q)} K_{\sigma, \mu}^{(q)} \widetilde{L}_{\mu, \eta}^{(q)} L_{\eta, \omega}^{\dagger(p, q) T}
$$

By substituting (9.4) in this expression, we obtain the identity

$$
L_{\tau, \eta}^{\dagger(p, q)}=K_{\tau, \eta}^{\dagger(p, q)}
$$

that equates the two transformation matrices. 
Such a simple result does not in general follow from (8.29) because the transformation

$$
Y_{a \lambda, b \eta}^{(p)}=\sum_{g, h \neq q} K_{\lambda, \omega}^{\dagger(p, q)} M_{a, g}^{(p, q)} Y_{g \omega, h \tau}^{(q)} M_{h, b}^{(p, q) T} K_{\tau, \eta}^{\dagger(p, q) T}
$$

satisfied by the matrix $Y$ is not an orthogonal transformation for composites with more than two components because $M$ is not an orthogonal matrix. Nevertheless, (8.29), (9.7), and (9.9) imply

$$
\hat{Y}_{a \lambda, b \eta}^{(p)}=\sum_{g \neq q} K_{\lambda, \omega}^{\dagger(p, q)} M_{a, g}^{(p, q)} \widehat{Y}_{g \omega, \tau}^{(q)} K_{\tau, b \eta}^{\dagger(p, q) T}
$$

and by comparing this with (9.10) we deduce

$$
K^{\dagger(j-1)} M^{(1)} Y^{(j)} M^{(1) T} K^{\dagger(j-1) T}=K^{\dagger(j)} \hat{Y}^{(j)} M^{(1) T} M^{(1)} \hat{Y}^{(j)} K^{\dagger(j) T},
$$

where $K^{\dagger(j)}, M^{(1)}$, and $Y^{(j)}$ are those matrices with elements $K_{\lambda, \omega}^{\dagger(p, q)}, M_{a, b}^{(p, q)}, Y_{\lambda, \omega}^{(q)}$ such that $o(\lambda)=o(\omega)=j$. Hence $K^{\dagger(j)}$ is that orthogonal matrix which transforms

$$
\hat{\gamma}^{(j)} M^{(1) T} M^{(1)} \hat{\gamma}^{(j)} \quad \text { into } K^{\dagger(j-1)} M^{(1)} Y^{(j)} M^{(1) T} K^{\dagger(j-1) T}
$$

Assuming the eigenvalues of $\hat{\gamma}^{(j)} M^{(1) T} \hat{\gamma}^{(j)}$ do not happen to be degenerate, this relation is sufficient to specify the matrix $K^{\dagger(j)}$ given $K^{\dagger(j-1)}$. By induction on $j$ (and recalling that $K^{\dagger(0)}=I^{(0)}$ ) we can thereby determine the transformation matrix $K^{\dagger}$ without reference to the intermediate transformations $K_{\tau, \eta}^{(p)}$ and $K_{\lambda, \omega}^{(q)}$. It is thus apparent that $K^{\dagger}$ depends on the weights but not on the normalization factors.

By substituting (9.3) and (9.9) in (8.7) we find that the canonical basis set $\mathbf{x}_{\omega}^{(q)}$ and $\mathbf{y}_{\tau}^{(q)}$ transforms into the new canonical basis set

$$
\mathbf{x}_{\eta}^{(p)}=K_{\eta, \omega}^{\dagger(p, q)} \mathbf{x}_{\omega}^{(q)}, \quad \mathbf{y}_{\lambda}^{(p)}=K_{\lambda, \tau}^{\dagger(p, q)} \mathbf{y}_{\tau}^{(q)}
$$

via the transformation matrix $K^{\dagger}$.

Aside from switches in sign the matrix $M$ for a two-component composite merely acts to interchange the component indices 1 and 2 and is obviously orthogonal. Hence the transformation (9.10) for $\hat{\gamma}$ is orthogonal and implies a similar transformation law for $Y$ which with (9.11) implies

$$
K_{a \omega, b \eta}^{\dagger(p, q)}=M_{a, b}^{(p, q)} K_{\omega, \eta}^{\dagger(p, q)} \text { for } \quad n=2 .
$$

This, in conjunction with (9.4) and (9.9), completely specifies the matrices $L^{\dagger}$ and $K^{\dagger}$ : by induction we deduce that

$$
L^{\dagger}=K^{\dagger}=M \quad \text { when } \quad n=2 .
$$

Consequently, for two-component composites, the fundamental geometric matrices $W_{a}$ and $N$ remain invariant under a change of reference medium, apart from a relabelling of indices.

\section{Geometric Matrices for Two-Dimensional Composites}

On the basis of a phase-interchange relationship due to Keller [38], Dykhne [39], and Mendelson [40] it has been established [12] that the normalization factors are unity for any two-dimensional, two-component isotropic composite. Here we 
show this property extends to multicomponent isotropic composites. In addition we establish that two-dimensional anisotropic, two-component composites have isotropic weights and have normalization factors with unit determinant.

Let $\Phi$ be the transformation that maps any two-dimensional field $\mathbf{P}=\left(P_{1}, P_{2}\right)$ into its orthogonal counterpart,

$$
\Phi \mathbf{P}=\left(-P_{2}, P_{1}\right)
$$

Now for any given polarization field $\mathbf{P}$, the associated current field $\mathbf{J}=\Gamma_{2} \mathbf{P}$ satisfies

$$
\nabla \cdot \mathbf{J}=0, \quad \nabla \times(\mathbf{J}-\mathbf{P})=0, \quad \Gamma_{0} \mathbf{J}=0,
$$

and consequently we have

$$
\nabla \times \Phi \mathbf{J}=0, \quad \nabla \cdot(\Phi \mathbf{J}-\Phi \mathbf{P})=0, \quad \Gamma_{0} \Phi \mathbf{J}=0,
$$

which implies $\Phi \mathbf{J}=\Gamma_{1} \Phi \mathbf{P}$, and subsequently that

$$
\Gamma_{1} \Phi=\Phi \Gamma_{2} .
$$

This property of the two-dimensional operator $\Phi$ has been discussed in detail by Mendelson [40] and Kohler and Papanicolaou [24] and provides the key step in proving the phase-interchange relationship.

Now since $\chi_{a}$ and $\Phi$ commute,

$$
\chi_{a} \Phi=\Phi \chi_{a}
$$

for all components $a$, the fields $\mathbf{J}_{\tau}, \mathbf{E}_{\tau}$, and $\mathbf{P}_{a \tau}$ defined in Sect. 4 must from (10.4) satisfy

$$
\begin{gathered}
\Phi \mathbf{J}_{\alpha 1}=-(-1)^{o(\alpha)} \mathbf{E}_{\alpha 2}, \quad \Phi \mathbf{J}_{\alpha 2}=(-1)^{o(\alpha)} \mathbf{E}_{\alpha 1}, \\
\Phi \mathbf{P}_{a \alpha 1}=(-1)^{o(\alpha)} \mathbf{P}_{a \alpha 2}, \quad \Phi \mathbf{P}_{a \alpha 2}=-(-1)^{o(\alpha)} \mathbf{P}_{a \alpha 1},
\end{gathered}
$$

for all strings, $\alpha$, of component indices. This implies the identity

$$
V_{\alpha 1, \beta 1}^{(q)} \equiv\left\langle\mathbf{J}_{\alpha 1}^{(q)} \mid \mathbf{J}_{\beta 1}^{(q)}\right\rangle=\left\langle\Phi \mathbf{J}_{\alpha 1}^{(q)} \mid \Phi \mathbf{J}_{\beta 1}^{(q)}\right\rangle=\left\langle\mathbf{E}_{\alpha 1}^{(q)} \mid \mathbf{E}_{\beta 1}^{(q)}\right\rangle \equiv U_{\alpha 2, \beta 2}^{(q)},
$$

and similarly we have

$$
V_{\alpha 2, \beta 2}^{(q)}=U_{\alpha 1, \beta 1}^{(q)}, \quad Z_{a, \alpha 1, \beta 1}^{(q)}=Z_{a, \alpha 2, \beta 2}^{(q)}, \quad V_{\alpha 1, \beta 2}^{(q)}=-U_{\alpha 2, \beta 1}^{(q)}, \quad V_{\alpha 2, \beta 1}^{(q)}=-U_{\alpha 1, \beta 2}^{(q)} .
$$

These relations are, of course, consistent with (5.4) and (5.6). Next, by noting that the inner product of any vector with its orthogonal counterpart is zero, we deduce

$$
\left\langle c_{1} \mathbf{P}_{a \alpha 1}+c_{2} \mathbf{P}_{a \beta 1}|\Phi| c_{1} \mathbf{P}_{a \alpha 1}+c_{2} \mathbf{P}_{a \beta 1}\right\rangle=0
$$

for all real constants $c_{1}$ and $c_{2}$ and thereby conclude that

$$
Z_{a, \alpha 1, \beta 2}^{(q)}=-Z_{a, \alpha 2, \beta 1}^{(q)} \text {. }
$$

For an isotropic material the identities (10.7) and (10.8) imply $U=V$ and from (8.19) and (8.20) we see that this can only occur when

$$
N=I \text {. }
$$

Thus for any two-dimensional isotropic composite the normalization factor is simply the identity matrix, and therefore all the matrix elements (8.31) and (8.32) of the operators $\Gamma_{1}$ and $\Gamma_{2}$ are completely determined in the canonical basis set. 
For a two-component anisotropic composite the strings $\alpha, \beta$ in (10.7)-(10.10) represent either a chain of 1's if $q=2$ or a chain of 2's if $q=1$. If $\alpha$ and $\beta$ have the same length, then $\alpha=\beta$. Consequently (10.8) and (10.10) together with (7.5) imply that the matrices $Z_{a}^{(j)}, H^{(j)}$, and $G^{(j)}$ are multiples of the identity matrix $\boldsymbol{}^{(j)}$. By applying the principle of induction, it follows from $(8.12)-(8.17)$ that $K^{(j)}, L^{(j)}, W_{a}^{(j)}$, and $N^{(j)}+\left(N^{(j)}\right)^{-1}$ are likewise multiples of $/^{(j)}$. In addition $\widetilde{U}$ and $\widetilde{V}$, given by $(8.3)$ and (8.4), must satisfy relations directly analogous to the relations (10.7) and (10.8) satisfied by $U$ and $V$. Hence each normalization factor $N^{(j)}, j=1,2, \ldots, \infty$, must have unit determinant.

Thus, for any two-dimensional, two-component anisotropic composite the weights $W_{1}^{(j)}, W_{2}^{(j)}, j=0,1, \ldots, \infty$, are sequences of isotropic $2 \times 2$ matrices expressible in terms of positive scalar weights $w_{1}^{(j)}=1-w_{2}^{(j)}$ through the equation

$$
W_{a}^{(j)}=w_{a}^{(j)} /^{(j)} \quad \text { for } \quad a=1,2,
$$

while the normalization factors $N^{(j)}, j=1,2, \ldots, \infty$, form a sequence of positive definite $2 \times 2$ matrices each with unit determinant. This extends earlier work of Golden [41] who obtained some elementary relations amongst the geometric parameters of anisotropic two-dimensional composites.

In summary, we have found simple constraints on the normalization factors and weights of two-dimensional isotropic multicomponent composites and twodimensional anisotropic two-component composites. The question of what (10.8) and (10.10) imply about the fundamental geometric parameters of anisotropic multicomponent two-dimensional composites is left unresolved.

\section{The Continued Fraction Representation for $\sigma^{*}$}

In the first part of this section we demonstrate how a continued fraction representation for $\sigma^{*}$ can be developed from the Dirichlet variational definition of the effective conductivity tensor,

$$
\left\langle\mathbf{e}\left|\sigma^{*}\right| \mathbf{e}\right\rangle \equiv \min _{\mathbf{E} \in \mathscr{E}}\left\langle\mathbf{e}+\mathbf{E}\left|\sum_{a=1}^{n} \sigma_{a} \chi_{a}\right| \mathbf{e}+\mathbf{E}\right\rangle,
$$

which holds when the variables $\sigma_{a}, a=1,2, \ldots, n$, are real and positive: see Synge [41]. Although this method sheds light on the connection between the continued fraction representation and well-known bounds on $\sigma^{*}$, it is not the simplest approach. As we will see in the second part of this section, the continued fraction expansion can be obtained directly by using the field equation recursion method, without resort to variational principles and without assuming the component conductivities are real. In the remainder of the section we show how the expansion simplifies for two-component composites.

\subsection{The Derivation from Variational Principles}

The Lax-Milgram lemma [28] implies that the minimum in (11.1) is attained within the Hilbert space $\mathscr{E}$ spanned by the fields $\mathbf{E}_{\tau}^{\prime \prime}$ and so, without loss of generality, we can restrict attention to fields $\mathbf{E}$ of the form

$$
\mathbf{E}=\sum_{\tau} \alpha_{\tau} \mathbf{E}_{\tau}^{\prime \prime(q)},
$$


where sum extends over all strings $\tau$ of indices not containing the exponent index $q$. Substituting this in (11.1) yields the expression

$$
\begin{aligned}
\mathbf{e}^{T} \sigma^{*} \mathbf{e}= & \min _{\alpha}\left[\sum_{a=1}^{n} \sigma_{a} e_{\ell}\left\langle\mathbf{x}_{\ell}\left|\chi_{a}\right| \mathbf{x}_{k}\right\rangle e_{k}+2 \sum_{a \neq q} \delta \sigma_{a} e_{k}\left\langle\mathbf{x}_{k}\left|\chi_{a}\right| \mathbf{E}_{\omega}^{\prime \prime(q)}\right\rangle \alpha_{\omega}\right. \\
& \left.+\alpha_{\tau}\left(\sigma_{q}\left\langle\mathbf{E}_{\tau}^{\prime \prime(q)} \mid \mathbf{E}_{\omega}^{\prime \prime(q)}\right\rangle+\sum_{a \neq q} \delta \sigma_{a}\left\langle\mathbf{E}_{\tau}^{\prime \prime(q)}\left|\chi_{a}\right| \mathbf{E}_{\omega}^{\prime \prime(q)}\right\rangle\right) \alpha_{\omega}\right],
\end{aligned}
$$

which from (8.37) and (8.31)-(8.33) can be re-expressed in the form

where

$$
\begin{aligned}
\mathbf{e}^{T} \sigma^{*} \mathbf{e}= & \min _{\alpha}\left\{\sum_{a=1}^{n}\left(\sigma_{a} \mathbf{e}^{T} W_{a} \mathbf{e}+2 \delta \sigma_{a} \mathbf{e}^{T} Y_{a} \boldsymbol{\alpha}\right)\right. \\
& \left.+\alpha^{T}\left[\Theta+\sum_{a=1}^{n}\left(\sigma_{a} \hat{N} W_{a} \hat{N}+2 \delta \sigma_{a} \hat{Y}_{a}^{T} \hat{N}\right)\right] \alpha\right\},
\end{aligned}
$$

$$
\Theta \equiv \sigma_{q} I+\sum_{a \neq q} \delta \sigma_{a} \hat{Y}_{a}^{T} \tilde{W}_{a} \hat{Y}_{a}
$$

is a block-diagonal matrix and $\hat{Y}_{a}$, with transpose $\hat{Y}_{a}^{T}$, is that submatrix of $Y$ with elements $Y_{a \tau}^{(q)} \omega$.

Now define $\alpha^{(j)}, j=1,2, \ldots, \infty$, as that subvector of $\alpha$ with elements $\alpha_{\tau}$ for which $o(\tau)=j$. Similarly let $W_{a}^{(j)}, \hat{N}^{(j)}, \hat{Y}_{a}^{(j)}$, and $\Theta^{(j)}$ denote those submatrices of $W_{a}, \widehat{N}, \hat{Y}_{a}$, and $\Theta$ with elements $W_{a, \tau, \omega}^{(q)}, \hat{N}_{\tau, \omega}^{(q)}, \hat{Y}_{a \lambda, \omega}^{(q)}$, and $\Theta_{\partial}^{(q)}, \omega$ such that $o(\tau)=o(\omega)$ $=1+o(\lambda)=j$. With these definitions (11.4) implies

$$
\begin{aligned}
\mathbf{e}^{T} \sigma^{*} \mathbf{e}= & \min _{\alpha} \lim _{h \rightarrow \infty}\left[\sum_{a=1}^{n} \mathbf{e}^{T} \sigma_{a} W_{a}^{(0)} \mathbf{e}+\sum_{j=1}^{h} \boldsymbol{\alpha}^{(j) T}\left(\Theta^{(j)}+\sum_{a=1}^{n} \sigma_{a} \widehat{N}^{(j)} W_{a}^{(j)} \widehat{N}^{(j)}\right) \boldsymbol{\alpha}^{(j)}\right. \\
& \left.+2 \sum_{j=2}^{h} \boldsymbol{\alpha}^{(j) T}\left(\sum_{a \neq q} \delta \sigma_{a} \hat{Y}_{a}^{(j) T}\right) \hat{N}^{(j-1)} \boldsymbol{\alpha}^{(j-1)}+2 \boldsymbol{\alpha}^{(1) T}\left(\sum_{a \neq q} \delta \sigma_{a} \hat{Y}_{a}^{(1) T}\right) \mathbf{e}\right] .
\end{aligned}
$$

Thus $\boldsymbol{\alpha}^{(j)}$ only couples with itself, $\boldsymbol{\alpha}^{(j+1)}$ or $\boldsymbol{\alpha}^{(j-1)}$ (or $\mathbf{e}$ if $j=1$ ), which is why a continued fraction expansion for $\sigma^{*}$ can be developed.

We now assume the operations of taking the limit $h \rightarrow \infty$ and the minimum over $\boldsymbol{\alpha}$ in (11.6) can be interchanged. By differentiating (11.6) it is evident that the optimum choice for $\boldsymbol{\alpha}^{(h)}$ is

where

$$
\boldsymbol{\alpha}^{(h)}=\left(\Theta^{(h)}+\hat{N}^{(h)} \Omega^{(h)} \hat{N}^{(h)}\right)^{-1}\left(\sum_{a=q} \delta \sigma_{a} \hat{Y}_{a}^{(h) T}\right) \hat{N}^{(h-1)} \boldsymbol{\alpha}^{(h-1)},
$$

$$
\Omega^{(h)} \equiv \sum_{a=1}^{n} \sigma_{a} W_{a}^{(h)}
$$

To repeat this procedure define, by recursion, the sequence of tensors

$$
\Omega^{(j-1)}=\sum_{a=1}^{n} \sigma_{a} W_{a}^{(j-1)}-\sum_{a, b \neq q} \delta \sigma_{a} \hat{\gamma}_{a}^{(j)}\left[\Theta^{(j)}+\hat{N}^{(j)} \Omega^{(j)} \hat{N}^{(j)}\right]^{-1} \hat{\gamma}_{b}^{(j) T} \delta \sigma_{b}
$$

for $j=h, h-1, h-2, \ldots, 1$, where $Y$ and $\Theta$ are defined via (8.26) and (11.5). (Note that $\Omega^{(j)} \equiv{ }^{h} \Omega^{(j)}$ depends on the truncation integer $h$.) After substituting (11.7) back 
into (11.6) and minimizing over $\boldsymbol{\alpha}^{(h-1)}, \boldsymbol{\alpha}^{(h-2)}, \ldots, \boldsymbol{\alpha}^{(1)}$, giving

$$
\boldsymbol{\alpha}^{(j)}=\left(\Theta^{(j)}+\hat{N}^{(j)} \Omega^{(j)} \hat{N}^{(j)}\right)^{-1}\left(\sum_{a \neq q} \delta \sigma_{a} \hat{Y}_{a}^{(j) T}\right) \hat{N}^{(j-1)} \boldsymbol{\alpha}^{(j-1)}
$$

for $j>1$, and

$$
\boldsymbol{\alpha}^{(1)}=\left(\Theta^{(1)}+\widehat{N}^{(1)} \Omega^{(1)} \widehat{N}^{(1)}\right)^{-1}\left(\sum_{a \neq q} \delta \sigma_{q} \hat{Y}_{a}^{(1) T}\right) \mathbf{e}
$$

we finally obtain the identity

$$
\sigma^{*}=\Omega^{(0)} \quad(\text { as } h \rightarrow \infty) .
$$

A continued fraction representation for $\sigma^{*}$ results when (11.9), or its equivalent form

$$
\begin{aligned}
\Omega^{(j-1)}= & \sum_{a=1}^{n} \sigma_{a} W_{a}^{(j-1)}-\sum_{a, b \neq q} \delta \sigma_{a}{ }_{a}^{(j)} \\
& \left.\times\left[\sigma_{q} \tilde{Y}^{(j)}+\sum_{c \neq q} \delta \sigma_{c} l_{c}^{(j) T} \tilde{W}_{c}^{(j-1)}\right)_{c}^{(j)}+\dot{Y}^{(j)} \widehat{N}^{(j)} \Omega^{(j)} \widehat{N}^{(j)} \dot{Y}^{(j)}\right]^{-1} I_{b}^{(j) T} \delta \sigma_{b}
\end{aligned}
$$

is repeatedly inserted in (11.12): here $I_{a}^{(j)}$ denotes the submatrix of the identity matrix $I^{(j)}$ that has elements $I_{a \lambda, \omega}=\delta_{a \lambda, \omega}$ with $o(\omega)=1+o(\lambda)=j$. When $h$ is kept finite, (11.6) implies

$$
\sigma^{*} \leqq \Omega^{(0)} \quad(h \text { finite })
$$

and thus the continued fraction expansion, terminated by (11.8), forms an upper bound on the effective conductivity $\sigma *$. We call such a bound a Wiener-Beran upper bound of order $2 h+1$, in recognition of their pioneering work $[17,18]$ on bounds of this type: the bounds incorporate information about the composite contained in $(2 h+1)$-point correlation functions. In fact the overall procedure outlined in this section [starting from the Dirichlet variational principles and using trail fields of the form (11.2)] is an extension of earlier collaborative work with Phan-Thien [22] in which the general bounding method of Beran [31] was implemented to obtain third-order bounds on multicomponent composites: see also the related work of Kroner [20] and Willis [21], among others.

Note that the $(2 h-1)$ th-order Wiener-Beran upper bounds necessarily get tighter as $h$ increases because our set of trail fields includes more and more fields. Hence the matrices $\Omega^{(0)} \equiv{ }^{h} \Omega^{(0)}, h=0,1,2, \ldots, \infty$, form a decreasing sequence. Since they are bounded below by $\sigma^{*}$ the limiting matrix ${ }^{\infty} \Omega^{(0)}$ must exist, as implicitly assumed in (11.12). The question of interchangeability of the minimum over $\alpha$ and the limit $h \rightarrow \infty$ in (11.6) therefore hinges on whether the $(2 h-1)$ th-order WienerBeran upper bound converges to $\sigma^{*}$ in the limit $h \rightarrow \infty$. This convergence will in fact be established in Sect. 7 of Part II, thereby providing a rigorous justification of (11.12).

\subsection{Derivation from the Field Equation Recursion Method}

The tensors ${ }^{h} \Omega_{\tau, \omega}^{(q, j)}$ have a very simple interpretation in the limit $h \rightarrow \infty$. Since their continued fraction expansion is exactly of the same form as the continued fraction expansion for $\sigma^{*}$, the matrix $\Omega^{(j)}\left(\sigma_{1}, \sigma_{2}, \ldots, \sigma_{n}\right)$ must be the effective tensor in the 
Hilbert space

$$
\mathscr{H}^{(j)}=\mathscr{U}^{(j)} \oplus \mathscr{E}^{(j)} \oplus \mathscr{J}^{(j)}=\mathscr{P}_{1}^{(j)} \oplus \mathscr{P}_{2}^{(j)} \oplus \ldots \oplus \mathscr{P}_{n}^{(j)}
$$

that is associated with the truncated set of geometric parameters $W_{a}^{(g)}$ and $N^{(g+1)}$ with $g \geqq j$.

To prove this directly, thus by-passing the need to use variational principles, consider the Hilbert space $\mathscr{H}^{(1)}$. From the structure of the matrices $(8.31),(8.32)$, and (8.33) representing $\Gamma_{1}, \Gamma_{2}$, and $\chi_{a}$ in the canonical basis set (see Figs. 1 and 2) we deduce that $\mathscr{H}^{(1)}$ is a subspace of $\mathscr{H}$, namely the orthogonal complement of the $n d$ dimensional subspace spanned by the orthonormal fields $\mathbf{x}_{\ell}$ and $\mathbf{y}_{a \ell}^{(q)}, \ell=1,2, \ldots, d$, $a=1,2, \ldots, q-1, q+1, \ldots, n$. The $d(n-1)$-dimensional space $\mathscr{U}^{(1)}$ is spanned by the fields $\mathbf{x}_{a \ell}^{(q)}$ while $\mathscr{E}^{(1)}=\mathscr{E} \cap \mathscr{H}^{(1)}$ and $\mathscr{J}^{(1)}=\mathscr{J} \cap \mathscr{H}^{(1)}$, respectively, are spanned by the fields $\mathbf{E}_{\eta}^{(q)}$ and $\mathbf{J}_{\eta}^{(q)}$ (or $\mathbf{E}_{\eta}^{(q)}$ and $\mathbf{J}_{\eta}^{(q)}$ ) with $o(\eta) \geqq 2$. The component Hilbert spaces $\mathscr{P}_{a}^{(1)}=\mathscr{P}_{a} \cap \mathscr{H}^{(1)}, a=1,2, \ldots, n$, are spanned by the fields $\mathbf{P}_{a \tau}^{(q)}\left(\right.$ or $\left.\mathbf{P}_{a \tau}^{(q)}\right)$ with $o(\tau) \geqq 1$. Since the canonical basis set satisfies the transformation (9.13) under a change of reference medium (where $K^{\dagger}$ is block-diagonal) it follows that the Hilbert spaces $\mathscr{H}^{(1)}, \mathscr{U}^{(1)}, \mathscr{E}^{(1)}, \mathscr{J}^{(1)}$, and $\mathscr{P}_{a}^{(1)}, a=1,2, \ldots, n$, are in fact all independent of our choice of reference medium, $q$.

In the canonical basis set the matrices representing the projection operators $\Gamma_{1}^{(1)}, \Gamma_{2}^{(1)}$, and $\chi_{a}^{(1)}$ that project onto $\mathscr{E}^{(1)}, \mathscr{J}^{(1)}$, and $\mathscr{P}_{a}^{(1)}$ are obtained from the matrices representing $\Gamma_{1}, \Gamma_{2}$, and $\chi_{a}$ by setting to zero those blocks of elements that incorporate $N^{(1)}$ and $W_{a}^{(0)}$ : see Figs. 1 and 2. Consequently from (9.33) we have

$$
\begin{aligned}
\sum_{a=1}^{n} \sigma_{a} \chi_{a}= & \sum_{a=1}^{n} \sigma_{a} \chi_{a}^{(1)}+\sum_{a=1}^{n} \sigma_{a} W_{a, \ell, k}^{(q)}\left|x_{\ell}\right\rangle\left\langle x_{k}\right| \\
& +\sum_{a, b \neq q} \delta \sigma_{a} Y_{a \ell, b \ell}^{(q)}\left(\left|\mathbf{y}_{b \ell}^{(q)}\right\rangle\left\langle\mathbf{x}_{\ell}|+| \mathbf{x}_{\ell}\right\rangle\left\langle\mathbf{y}_{b \ell}^{(q)}\right|\right) \\
& +\sum_{a, b \neq q} \Theta_{a \ell, b \ell}^{(q, 1)}\left|\mathbf{y}_{a \ell}^{(q)}\right\rangle\left\langle\mathbf{y}_{b \ell}^{(q)}\right|,
\end{aligned}
$$

where the $\Theta_{a \ell, b k}^{(q, 1)}$ are elements of the matrix $\Theta^{(1)}$ defined via (11.5).

Now suppose the field equation

$$
\left|\mathbf{j}^{(1)}+\mathbf{J}^{*(1)}\left(\mathbf{j}^{(1)}\right)\right\rangle=\sum_{a=1}^{n} \sigma_{a} \chi_{a}^{(1)}\left|\mathbf{e}^{(1)}+\mathbf{E}^{*(1)}\left(\mathbf{e}^{(1)}\right)\right\rangle
$$

is solved for $\mathbf{j}^{(1)} \in \mathscr{U}^{(1)}, \mathbf{J}^{*(1)}\left(\mathbf{j}^{(1)}\right) \in \mathscr{J}^{(1)}$, and $\mathbf{E}^{*(1)}\left(\mathbf{e}^{(1)}\right) \in \mathscr{E}^{(1)}$ as a function of $\mathbf{e}^{(1)} \in \mathscr{U}^{(1)}$. Let us define $\Omega^{(1)}$ as the associated effective tensor relating $\mathbf{j}^{(1)}$ and $\mathbf{e}^{(1)}$ via

$$
\mathbf{j}^{(1)}=\Omega^{(1)} \mathbf{e}^{(1)} .
$$

Of course, it remains to show that this definition is consistent with (11.9).

Given any uniform field $\mathbf{e} \in \mathscr{U}$, with components $e_{\ell} \equiv\left\langle\mathbf{e} \mid \mathbf{x}_{\ell}\right\rangle$, we seek to find some field $\mathbf{e}^{(1)}=\mathbf{e}^{(1)}(\mathbf{e}) \in \mathscr{U}^{(1)}$, with components $e_{a \ell}^{(q)}=\left\langle\mathbf{e}^{(1)} \mid \mathbf{x}_{a \ell}^{(q)}\right\rangle$, such that

$$
\mathbf{E}^{*}=\mathbf{E}^{*(1)}\left(\mathbf{e}^{(1)}\right)+\mathbf{e}^{(1)}+\sum_{a, b \neq q} e_{a \ell}^{(q)} \dot{N}_{a \ell, b k}^{(q)} \mathbf{y}_{b \ell}^{(q)},
$$

is the solution of the field equation (3.1). Note that the last term in this expression ensures, via (8.37), that $\mathbf{E}^{*} \in \mathscr{E}$. From (11.16)-(11.19) we have

$$
\begin{aligned}
\Gamma_{1} & \sum_{a=1}^{n} \sigma_{a} \chi_{a}\left|\mathbf{e}+\mathbf{E}^{*}\right\rangle \\
& =\Gamma_{1} \sum_{a, b \neq q}\left[\left|\mathbf{x}_{a \ell}^{(q)}\right\rangle \Omega_{a \ell, b \ell}^{(q, 1)} e_{b \ell}^{(q)}+\left|\mathbf{y}_{a \ell}^{(q)}\right\rangle\left(Y_{a \ell, b \ell}^{(q)} \delta \sigma_{b} e_{k}+\sum_{c \neq q} \Theta_{a \ell, c m}^{(q)} \dot{N}_{c m, b \ell}^{(q)} e_{b \ell}^{(q)}\right)\right]
\end{aligned}
$$


which, according to (8.37), vanishes when we set

$$
\mathbf{e}^{(1)}=-\widehat{N}^{(j)}\left(\Theta^{(1)}+\widehat{N}^{(1)} \Omega^{(1)} \widehat{N}^{(1)}\right)^{-1} \sum_{b \neq q} \delta \sigma_{b} Y_{b}^{T} \mathbf{e} .
$$

The field equation (2.11) is thereby solved and (11.19) implies

$$
\begin{aligned}
|\mathbf{j}\rangle & =\Gamma_{0} \sum_{a=1}^{n} \sigma_{a} \chi_{a}\left|\mathbf{e}+\mathbf{E}^{*}\right\rangle \\
& =\sum_{a=1}^{n} \sigma_{a} W_{a}|\mathbf{e}\rangle+\sum_{a \neq q}\left|\mathbf{x}_{\ell}\right\rangle \delta \sigma_{a} \mathbf{Y}_{a \ell} \dot{N}^{(1)} \mathbf{e}^{(1)},
\end{aligned}
$$

where $\mathbf{Y}_{a \ell}$ is that row of the matrix $Y$ with elements $Y_{a \ell}^{(q)}, b \AA$. By substituting (11.21) into (11.22) we conclude that $\sigma^{*}=\Omega^{(0)}$ is indeed given in terms of the conductivity matrix $\Omega^{(1)}$ via the relation (11.9). By repeating this argument and successively introducing the conductivity matrices $\Omega^{(j)}, j=2,3, \ldots, \infty$, we obtain the same continued fraction expansion for $\sigma^{*}$ as implied by (11.9) and (11.12). In contrast to the variational approach, this derivation of the continued fraction expansion applies even when the variables $\sigma_{a}, a=1,2, \ldots, n$, are complex.

Note from (11.18) and (9.13) that when the reference medium is changed from component $q$ to component $p$, the fundamental conductivity matrices $\Omega_{\tau, \omega}^{(p, j)}$ and $\Omega_{\eta, \lambda}^{(q, j)}$ are related via the orthogonal transformation

$$
\Omega_{\tau, \omega}^{(p, j)}=K_{\tau, \eta}^{\dagger(p, q)} \Omega_{\eta, \lambda}^{(q, j)} K_{\lambda, \omega}^{\dagger(p, q) T}
$$

and are both just different representations of the same effective conductivity tensor, $\Omega^{(j)}$, in $\mathscr{H}^{(j)}$.

Any matrix-valued function $\sigma_{\ell k}^{*}, \ell, k=1,2, \ldots, d$, that can be expressed as a continued fraction of the form implied by (11.12) and (11.9) for some allowable choice of normalization factors and weights will be denoted as an $n$-variable $\Omega$-function of rank $d$ and its associated continued fraction representation will be called an $\Omega$-fraction.

\subsection{The Recursion Relation for Two-Component Composites}

For a two-component isotropic composite the recursion relation (11.9) simplifies considerably since the weights, normalization factors, and conductivity matrices take the form

$$
W_{a}^{(j)}=w_{a}^{(j)} \boldsymbol{\prime}^{(j)}, \quad N^{(j)}=n^{(j)} \boldsymbol{\mu}^{(j)}, \quad \Omega^{(j)}=\sigma^{*(j)} \boldsymbol{\mu}^{(j)},
$$

where $w_{a}^{(j)}, n_{a}^{(j)}$, and $\sigma^{*(j)}$ are scalars with

$$
w_{1}^{(j)}+w_{2}^{(j)}=1 .
$$

The constants $w_{1}^{(0)}$ and $w_{2}^{(0)}$ can be identified via (8.22) with the volume fractions $f_{1}$ and $f_{2}$. Also (8.24) implies $n^{0}=d-1$ and $w_{1}^{(0)}$ and $w_{2}^{(0)}$ in fact correspond to the fundamental geometric parameters $\zeta_{1}$ and $\zeta_{2}$ introduced in [43], which in turn are related to a parameter $\lambda$ defined by Brown [29] that depends on the three-point correlation functions. These parameters $\zeta_{1}$ and $\zeta_{2}=1-\zeta_{1}$ have been accurately evaluated for cell-materials [43,44], for regular arrays of spheres and cylinders [45], and for random distributions of penetrable spheres and cylinders [46-48] 
and have been estimated for random distributions of impenetrable spheres [49, 50]. In fact, following earlier work of Corson [32], a procedure has recently been developed by Berryman [35] for calculating $\zeta_{1}$ directly via digital image processing techniques from a cross-sectional photograph of the microstructure. Ideally all this work should be extended to anisotropic two-component composites: such a scheme has already been initiated by Gillette [34].

By substituting (11.24) in (11.9) we obtain the recursion relation

$$
\sigma^{*(j-1)}=w_{1}^{(j-1)} \sigma_{1}+w_{2}^{(j-1)} \sigma_{2}-\frac{w_{1}^{(j-1)} w_{2}^{(j-1)}\left(\sigma_{1}-\sigma_{2}\right)^{2}}{w_{1}^{(j-1)} \sigma_{2}+w_{2}^{(j-1)} \sigma_{1}+n^{(j)} \sigma^{*(j)}}
$$

that agrees exactly with the expression [12] derived on the basis of the analytic properties of $\sigma^{*}\left(\sigma_{1}, \sigma_{2}\right)$, following the approach developed in Appendix 4 of Part II. The form of the recursion relation was originally motivated by the structure of simplified expressions for Beran's variational bounds [18] obtained independently by Torquato and Stell $[46,51]$ and Milton [43], and by work of Berryman [52] establishing a connection with Bruggeman's effective medium approximation. (This connection is developed further in Sect. 14.)

For an anisotropic two-dimensional, two-component composite the recursion relation again simplifies due to the special structure (10.12) of the weights and we have

$$
\begin{aligned}
\Omega^{(j-1)}= & \left(w_{1}^{(j-1)} \sigma_{1}+w_{2}^{(j-1)} \sigma_{2}\right) /^{(j-1)}-w_{1}^{(j-1)} w_{2}^{(j-1)}\left(\sigma_{1}-\sigma_{2}\right)^{2} \\
& \times\left[\left(w_{1}^{(j-1)} \sigma_{2}+w_{2}^{(j-1)} \sigma_{1}\right) /^{(j)}+\hat{N}^{(j)} \Omega^{(j)} \hat{N}^{(j)}\right]^{-1},
\end{aligned}
$$

where the $2 \times 2$ matrices $\hat{N}^{(j)}, j=1,2, \ldots, \infty$, have unit determinant.

\section{Equivalent Continued Fraction Representations}

The recursion relation (11.9) can be expressed in three other equivalent forms that help shed light on the analytic properties of $\Omega$-functions (see Part II). Let us introduce the variables

and the matrix

$$
\hat{s}_{a}=\hat{s}_{a}^{(q)}=\left(s_{a}^{(q)}\right)^{1 / 2}=\left[\sigma_{q} /\left(\sigma_{q}-\sigma_{a}\right)\right]^{1 / 2}
$$

$$
\Pi^{+(j)} \equiv\left(\tilde{\Pi}^{+(j)}\right)^{-1} \equiv I^{(j)}+\sigma_{q}^{-1} \widehat{N}^{(j)} \Omega^{(j)} \hat{N}^{(j)}
$$

By manipulating (11.13) we have

$$
\sigma_{q}^{-1} \Omega^{(j-1)}=I^{(j-1)}+\sum_{a, b \neq q} \hat{W}_{a}^{(j-1)} \tilde{\Delta}_{a, b}^{+(j)} \hat{W}_{b}^{(j-1)}
$$

where $\tilde{W}_{a}^{(j-1)}$ is the square root of $W_{a}^{(j-1)}$ and $\tilde{\Delta}^{+(j)}$ is the matrix defined by

$$
\begin{aligned}
\tilde{\Delta}_{a, b}^{+(j)} \equiv & \hat{s}_{a}\left\{-I_{a, b}^{(j)}+I_{a}^{(j)}\left[I^{(j)}-\sum_{c, d \neq q} I_{c}^{(j) T} \hat{S}_{c}^{-1} \hat{W}_{c}^{(j-1)} \dot{Y}_{c}^{(j)}\right.\right. \\
& \left.\left.\times \Pi^{+(j)} \dot{Y}_{d}^{(j) T} \hat{W}_{d}^{(j-1)} \hat{s}_{d}^{-1} I_{d}^{(j)}\right]^{-1} I_{b}^{(j) T}\right\} \hat{s}_{b}
\end{aligned}
$$

which is expressed in this cumbersome manner so its inverse

$$
\Delta^{+(j)} \equiv\left(\tilde{\Delta}^{+(j)}\right)^{-1}
$$


can be readily calculated using the identity

$$
(I-A)^{-1}-I=\left(A^{-1}-\right)^{-1}
$$

which holds for any symmetric matrix $A$. Specifically we find that

$$
\Delta_{a, b}^{+(j)}=\dot{W}_{a}^{(j-1)} \hat{\gamma}_{a}^{(j)} \tilde{\Pi}^{+(j)} \hat{Y}_{b}^{(j)} \dot{W}_{b}^{(j-1)}-s_{a}{ }_{a, b}^{(j)},
$$

where $\dot{W}_{a}^{(j-1)}$ denotes the inverse of $\hat{W}_{a}^{(j-1)}$, i.e. the square root of $\tilde{W}_{a}^{(j-1)}$.

Thus by inserting the definition (12.2) of $\Pi^{+(j)}$ in this formula and substituting the resulting expression for $\Delta^{+(j)}$ in (12.3) we obtain an alternative continued fraction expansion for $\sigma^{*}$. For a two-component isotropic composite the form of the resulting recursion relation is particularly simple: the expression

$$
\sigma^{*(j-1)} / \sigma_{2}=1+w_{1}^{(j-1)}\left\{-s_{1}+w_{2}^{(j-1)}\left[1+\left(n^{(j)} \sigma^{*(j)}\right) / \sigma_{a}\right]^{-1}\right\}^{-1}
$$

is, for example, obtained when $\sigma_{q}=\sigma_{2}$. A slightly different form of this continued fraction expansion (12.8) was derived by Golden [14, 41] and is essentially equivalent to the well-known expansion of Stieltjes functions as $S$-fractions $[1,2]$.

Some additional continued fraction representations are obtained by defining

$$
\tilde{\Pi}^{-(j)} \equiv\left(\Pi^{-(j)}\right)^{-1} \equiv /^{(j)}-\tilde{\Pi}^{+(j)},
$$

the matrix,

$$
\Delta_{a, b}^{-(j)} \equiv-\hat{W}_{a}^{(j-1)} \hat{W}_{b}^{(j-1)}-\Delta_{a, b}^{+(j)}
$$

and the inverse matrices

$$
\widetilde{\Omega}^{(j)} \equiv\left(\Omega^{(j)}\right)^{-1}, \quad \tilde{\Delta}^{-(j)} \equiv\left(\Delta^{-(j)}\right)^{-1},
$$

which, in fact, satisfy relations analogous to (11.9), (12.2), (12.3), and (12.7). To see this first note from (12.2) and (12.6) that the equation

$$
\Pi^{-(j)}=\boldsymbol{I}^{(j)}+\sigma_{q} \dot{N}^{(j)} \widetilde{\Omega}^{(j)} \dot{N}^{(j)}
$$

is satisfied. Second, by substituting (12.7) in (12.10) and using (12.9) and the defining equation (8.26) for $Y_{a, b}$ we deduce

$$
\Delta_{a, b}^{-(j)}=\dot{W}_{a}^{(j-1)} \hat{Y}_{a}^{(j)} \tilde{\Pi}^{-(j)} \hat{Y}_{b}^{(j) T} \dot{W}_{b}^{(j-1)}-t_{a} I_{a, b}^{(j)},
$$

in which $t_{a}=t_{a}^{(q)}$ is defined by (3.10). Third, the relation

$$
\sigma_{q} \widetilde{\Omega}^{(j-1)}=I^{(j-1)}+\sum_{a, b \neq q} \hat{W}_{a}^{(j-1)} \tilde{\Delta}_{a, b}^{-(j)} \hat{W}_{b}^{(j-1)}
$$

follows directly from (12.3) and a general property of matrices; namely that any symmetric matrix of the form

$$
D=A+\sum_{a, b \neq q} B_{a}^{T} \widetilde{C}_{a, b} B_{b}
$$

in which $A$ and $\widetilde{C}$ are symmetric matrices, has the inverse

where

$$
\widetilde{D}=A^{-1}+\sum_{a, b \neq q} A^{-1} B_{a}^{T} \widetilde{C}_{a, b}^{\prime} B_{b} A^{-1}
$$

$$
C_{a, b}^{\prime} \equiv-B_{a} A^{-1} B_{b}^{T}-C_{a, b}, \quad C \equiv(\widetilde{C})^{-1}, \quad C^{\prime} \equiv\left(\widetilde{C}^{\prime}\right)^{-1}
$$


This property can be checked by taking the product of (12.15) with (12.16) to obtain the result

$$
I=I+\sum_{a, d \neq q} B_{a}^{T}\left[\widetilde{C}_{a, b}+\sum_{b, c \neq q} \widetilde{C}_{a, b}\left(B_{b} A^{-1} B_{c}^{T}+C_{b, c}\right) \widetilde{C}_{c, d}^{\prime}\right] B_{d} A^{-1},
$$

which is obviously satisfied. These three relations (12.12)-(12.14) of course imply a continued fraction expansion analogous to the one that results from (12.2), (12.3), and (12.7). For a two-component isotropic composite this continued fraction takes the form implied by the recursion relation

$$
\sigma_{2} / \sigma^{*(j-1)}=1+w_{1}^{(j-1)}\left\{-t_{1}+w_{2}^{(j-1)}\left[1+\sigma_{2} /\left(n^{(j)} \sigma^{*(j)}\right)\right]^{-1}\right\}^{-1},
$$

when the choice $\sigma_{q}=\sigma_{2}$ is made.

Lastly, note that the steps in the argument leading to the expression (12.7) from (11.9), (12.2), and (12.3) can be reversed to obtain (11.9) from (12.2), (12.3), and (12.7). By similar analysis, and by defining

$$
\delta \tilde{\sigma}_{a} \equiv \delta \tilde{\sigma}_{a}^{(q)} \equiv \sigma_{a}^{-1}-\sigma_{q}^{-1}=-\sigma_{q}^{-1} t_{a}^{-1},
$$

the recursion relation

$$
\begin{aligned}
\tilde{\Omega}^{(j-1)}= & \sum_{a=1}^{n} \sigma_{a}^{-1} W_{a}^{(j-1)}-\sum_{a, b \neq q} \delta \tilde{\sigma}_{a} \hat{Y}_{a}^{(j)} \\
& \times\left[{ }^{(j)} / \sigma_{q}+\sum_{c \neq q} \delta \tilde{\sigma}_{c} \hat{Y}_{c}^{(j) T} \tilde{W}_{c}^{(j-1)} \varphi_{c}^{(j)}+\dot{N}^{(j)} \tilde{\Omega}^{(j)} \dot{N}^{(j)}\right]^{-1} \hat{Y}_{b}^{(j) T} \delta \tilde{\sigma}_{b}
\end{aligned}
$$

analogous to (11.9) is obtained from (12.12)-(12.14). For a two-component isotropic composite this expression simplifies and we find

$$
1 / \sigma^{*(j-1)}=w_{1}^{(j-1)} / \sigma_{1}+w_{2}^{(j-1)} / \sigma_{2}-\frac{w_{1}^{(j-1)} w_{2}^{(j-1)}\left(1 / \sigma_{1}-1 / \sigma_{2}\right)^{2}}{w_{1}^{(j-1)} / \sigma_{2}+w_{2}^{(j-1)} / \sigma_{1}+1 /\left(n^{(j)} \sigma^{*(j)}\right)^{.}}
$$

These recursion relations (12.21) and (12.22), and the associated continued fraction expansions of $\sigma^{*}$, can alternatively be derived from the Thomson variational principle,

$$
\left\langle\mathbf{j}\left|\left(\sigma^{*}\right)^{-1}\right| \mathbf{j}\right\rangle=\min _{\mathbf{J} \in \mathscr{J}}\left\langle\mathbf{j}+\mathbf{J}\left|\sigma^{-1}\right| \mathbf{j}+\mathbf{J}\right\rangle
$$

by following essentially the same steps which led to (11.9) from the Dirichlet variational principle (11.1). If, in this approach, $h$ is kept finite and $\Omega^{(h)}$ is redefined through the equation

$$
\Omega^{(h)}=\left(\sum_{a=1}^{n} W_{a}^{(h)} / \sigma_{a}\right)^{-1}
$$

then the continued fraction generated by (12.21) is found from the variational principle (12.23) to correspond to a lower bound on $\sigma^{*}$, denoted as a Wiener-Beran lower bound of order $2 h+1$.

Following similar analysis, given in Appendix 2, the continued fraction expansion that results from the supplementary recursion relations (12.2), (12.3), and (12.7) is obtainable from the Hashin-Shtrikman variational principles [19]. 
When $h$ is kept finite the Hashin-Shtrikman variational principles yield both upper and lower bounds on $\sigma^{*}$. The bounds are biased with respect to one of the components, say component $m$, and are generated by substituting

$$
\Omega^{(h)}=\sigma_{m} I^{(h)}
$$

in the recursion relations to obtain a terminating continued fraction expansion for $\Omega^{(0)}$ : this terminating continued fraction is an upper bound when $\sigma_{m} \geqq \sigma_{a}, \forall a$, and a lower bound when $\sigma_{m} \leqq \sigma_{a}, \forall a$. These bounds depend on the $2 h$-point correlation functions that characterize the geometry of the composite, and hence are denoted as Hashin-Shtrikman upper and lower bounds of order $2 h$.

Countless other continued fraction expansions can be found. For example, it is clear that by changing reference media from component $q$ to say component $p$, we obtain a completely different (but nonetheless equivalent) set of continued fraction representations for $\sigma^{*}$, in terms of the normalization factors $N_{\lambda, \eta}^{(p)}$ and the weights $W_{a, \lambda, \eta}^{(p)}$.

Alternatively (11.9) can be used to express $\sigma^{*}$ in terms of $\Omega^{(j)}$ using the reference medium $q$ and then a continued fraction expansion for $\Omega^{(j)}$ can be developed using the reference medium $p$. Many other continued fraction expansion for $\sigma^{*}$ that are variants of this approach can of course be derived.

\section{Terminating Continued Fractions}

Here we examine several conditions under which the continued fraction expansion (11.9) for $\sigma^{*}$ terminates at some level, $g$, in the hierarchy. For this to be possible, the matrices $U, V$, and $Z_{a}$ must be singular, in contrast with the assumption made in Sect. 5. Thus we are, in effect, exploring the consequences of relaxing this assumption. When the continued fraction terminates the effective conductivity $\sigma^{*}$ becomes a rational function of the component conductivities and the Hilbert space $\mathscr{H}$ has finite dimension. We find the Hashin-Shtrikman and Wiener-Beran bounds correspond to terminating continued fractions generated by particularly simple allowable sets of geometric parameters.

Let us, for simplicity, suppose that the weights $W_{a}^{(j-1)}$ and the normalization factors $N^{(j)}$ are non-singular for $j=1$ up to say $j=g$. Then the dependence of $\sigma^{*}$ on $\Omega^{(g+1)}$ vanishes if and only if the dependence of $\sigma^{(g)}$ on $\Omega^{(g+1)}$ vanishes. From (11.9), this obviously occurs when the matrix $Y^{(g+1)}$ vanishes; which according to (8.26) will happen if and only if the weights $W_{a}^{(g)}, a=1,2, \ldots, n$ share a common set of eigenvectors and each has a combination of zero and unit eigenvectors (see Fig. 3). No other eigenvalues are allowed. We call such a set of weights a terminating set because successive weights and normalization factors do not influence the effective conductivity: the continued fraction expansion terminates at the gth level in the hierarchy. When $\gamma^{(g+1)}$ vanishes, the expression (11.9) for $\Omega^{(g)}$ takes the simple form

$$
\Omega^{(g)}=\sum_{a=1}^{n} \sigma_{a} W_{a}^{(g)}=\left(\sum_{a=1}^{n} W_{a}^{(g)} / \sigma_{a}\right)^{-1},
$$

in which equivalence of the two expressions for $\Omega^{(g)}$ follows from the structure of the terminating set of weights, $W_{a}^{(g)}$. Thus, with this set of weights, the upper and 


$$
\underline{\underline{W}}_{1}^{(2)}=\left[\begin{array}{llll}
1 & 0 & 0 & 0 \\
0 & 0 & 0 & 0 \\
0 & 0 & 0 & 0 \\
0 & 0 & 0 & 1
\end{array}\right] \underline{\underline{W}}_{2}^{(2)}=\left[\begin{array}{llll}
0 & 0 & 0 & 0 \\
0 & 1 & 0 & 0 \\
0 & 0 & 0 & 0 \\
0 & 0 & 0 & 0
\end{array}\right] \underline{\underline{W}}_{3}^{(2)}=\left[\begin{array}{llll}
0 & 0 & 0 & 0 \\
0 & 0 & 0 & 0 \\
0 & 0 & 1 & 0 \\
0 & 0 & 0 & 0
\end{array}\right]
$$

(a)

$$
\underline{N}^{(2)}=\left[\begin{array}{cccc}
0 & 0 & 0 & 0 \\
0 & \infty & 0 & 0 \\
0 & 0 & 0 & 0 \\
0 & 0 & 0 & \infty
\end{array}\right]
$$

(b)

$$
\underline{N}^{(2)}=\left[\begin{array}{llll}
\infty & 0 & 0 & 0 \\
0 & \infty & 0 & 0 \\
0 & 0 & \infty & 0 \\
0 & 0 & 0 & \infty
\end{array}\right]
$$

(c)

Fig. 3. Example of a terminating set of weights (a) and two terminating normalization factors (b and c) for a field equation with $d=1$ and $n=3$. The figure is schematic in that the matrices need not be diagonal. For the normalization factor of $\mathbf{c}$ the 4th-order Hashin-Shtrikman upper and lower bounds coincide and equal the 3rd-order Wiener-Beran upper bound

lower Wiener-Beran bounds of order $2 h+1$, coincide and determinate the effective conductivity exactly; there is no need to calculate higher order correlation functions.

These terminating sets of weights, like other sets of terminating geometric parameters, have a special significance reflected in the structure of the Hilbert space $\mathscr{H}$. Since $\gamma^{(g+1)}$ is zero, (8.29) implies the transformation matrix $\tilde{L}^{(g+1)}$ is zero: $K^{(g)}$ is non-singular because it is determined by the lower order weights and normalization factors which, by our assumption, are non-singular. Ignoring mathematical technicalities this implies, via (8.1), that the fields $\mathbf{E}_{\eta}^{(q)}$ and $\mathbf{J}_{\eta}^{(q)}$ vanish when $o(\eta)=g+1$. All higher order fields, including the polarization fields $\mathbf{P}_{a \tau}^{(q)}$ with $o(\tau) \geqq g+1$, are likewise zero because they are defined through projections and repeated projections of these fields $\mathbf{E}_{\eta}^{(q)}$ and $\mathbf{J}_{\eta}^{(q)}$, with $o(\eta)=g+1$, onto various subspaces. Thus the Hilbert space $\mathscr{H}$ has finite dimension and is spanned by the fields (vectors) $\mathbf{x}_{\ell}, \mathbf{E}_{\eta}^{(q)}$, and $\mathbf{J}_{\eta}^{(q)}$ with $\ell=1,2, \ldots, d$ and $o(\eta) \leqq g$.

Of particular importance are the terminating continued fractions generated by choosing some component $m$ and taking

$$
W_{a}^{(g)}=0 \quad \text { for } \quad a \neq m \text { and } W_{m}^{(g)}=l,
$$

which implies, via (13.1), that

$$
\Omega^{(g)}=\sigma_{m} I^{(g)} .
$$

Thus, given any allowable set of non-singular geometric parameters, the HashinShtrikman bounds of order $2 g$ are obtained from the infinite continued fraction expansion for $\sigma^{*}$ by replacing the given weights $W_{a}^{(g)}$ with the terminating set (13.2). The Hashin-Shtrikman bounds therefore correspond to allowable sets of geometric parameters.

If the weights $W_{a}^{(g)}$ are all non-singular, then the dependence of $\Omega^{(g)}$ on $\Omega^{(g+1)}$ can still vanish provided the normalization factor $N^{(g+1)}$ has a mixture of zero and infinite eigenvalues, and no other eigenvalues (see Fig. 3). In this limit $\tilde{\Pi}^{+(g+1)}$ and $U^{\prime(g+1)}$ defined by (12.2) and (8.3) act as projection operators on the space spanned by those eigenvectors of $N^{(g+1)}$ that have zero eigenvalues, while $\tilde{\Pi}^{-(g+1)}$ and $V^{\prime(g+1)}$ defined by (12.9) and (8.4) project onto the complementary space, spanned by those eigenvectors of $N^{(g+1)}$ that have infinite eigenvalues. 
Consequently, the expressions (12.7) and (12.13) for the supplementary conductivity matrices take the form

$$
\begin{aligned}
& \Delta_{a, b}^{+(g+1)}=\dot{W}_{a}^{(g)} \hat{Y}_{a}^{(g+1)} U^{\prime(g+1)} \hat{Y}_{b}^{(g+1) T} \dot{W}_{b}^{(g)}-S_{a} l_{a, b}^{(g+1)}, \\
& \Delta_{a, b}^{-(g+1)}=\dot{W}_{a}^{(g)} \hat{Y}_{a}^{(g+1)} V^{(g+1)} \hat{Y}_{b}^{(g+1) T} \dot{W}_{b}^{(g)}-t_{a} l_{a, b}^{(g+1)},
\end{aligned}
$$

which with (12.3) and (12.14) give expressions for $\Omega^{(g)}$ in terms of the weights, $W_{a}^{(g)}$ and the eigenvectors of the terminating normalization factor $N^{(g+1)}$. Since the dependence of $\Omega^{(0)}$ on $\Omega^{(g+1)}$ has vanished for such $N^{(g+1)}$, the upper and lower Hashin-Shtrikman bounds of order $2 g+2$ must clearly coincide and equal the effective conductivity $\sigma^{*}$ exactly: again there is no need to calculate higher order correlation functions.

For this set of terminating normalization factors the transformation matrix $\widetilde{K}^{(g+1)}$ given by (8.28) is zero. This implies, via (8.1), that the Hilbert space $\mathscr{H}$ has finite dimension and is spanned by the fields $\mathbf{P}_{\tau}^{(q)}$ with $o(\tau) \leqq g+1$.

When $N^{(g+1)}$ vanishes entirely, i.e. has only zero eigenvalues, (13.4) implies

$$
\Delta_{a, b}^{-(g+1)}=-t_{a} I_{a, b}^{(g+1)},
$$

which leads, via (12.14), to the identity

$$
\Omega^{(g)}=\left(\sum_{a=1}^{n} W_{a}^{(g)} / \sigma_{a}\right)^{-1} .
$$

So, for any given set of non-singular geometric parameters, the Wiener-Beran lower bound of order $2 g+1$ is obtained from the infinite continued fraction expansion for $\sigma^{*}$ by replacing $N^{(g+1)}$ with the terminating normalization factor

$$
N^{(g+1)}=0 \text {. }
$$

Similarly, the Wiener-Beran upper bound of order $2 g+1$ is obtained by replacing the given $N^{(g+1)}$ with the complementary terminating normalization factor,

$$
N^{(g+1)}=\infty /^{(g+1)} .
$$

Thus the Wiener-Beran bounds, like the Hashin-Shtrikman bounds, correspond to allowable sets of geometric parameters.

The dependence of $\sigma^{*}$ on $\Omega^{(g+1)}$ vanishes under many other circumstances which we have not investigated. For example, the possibility that the weights $W_{a}^{(g)}$ and the normalization factor $N^{(g+1)}$ are both singular has not been explored.

\section{A Simple Approximation for $\sigma$ *}

One of the best known formulae for estimating the scalar effective conductivity, $\sigma^{*}$, of an isotropic composite is Bruggeman's effective medium approximation [23]

$$
\sum_{a=1}^{n} f_{a}\left(\sigma_{a}-\sigma^{*}\right)\left[\sigma_{a}+(d-1) \sigma^{*}\right]^{-1}=0
$$

that applies to a space-filling aggregate of grains, treated as spherical in the simplest approximation, with various conductivities $\sigma_{1}, \sigma_{2}, \ldots, \sigma_{n}$. This approximation, also known as the coherent potential approximation, is in fact realizable and 
has been rigorously shown [53] to correspond to a hierarchical model with a very wide distribution of sphere sizes, similar to an earlier self-similar model of Sen et al. [54]. For a review of effective medium approximations see Landauer [55], Watt et al. [56], Korringa [57], Willis [21], Berryman [58], and Niklasson and Granqvist [59].

Here we prove that the effective medium approximation formula (14.1) is obtained from the simple choice

$$
W_{a, \lambda, \eta}^{(q)}=f_{a} \delta_{\lambda \eta}, \quad N_{\lambda, \eta}^{(q)}=n^{0} \delta_{\lambda \eta}
$$

of fundamental geometric parameters, where we set

$$
n^{0}=(d-1)
$$

so that (8.24) is satisfied. This result extends earlier work of Berryman [52] who found that the effective medium approximation for two-component composites is obtained when one substitutes

$$
w_{1}^{(j-1)}=f_{1}, \quad w_{2}^{(j-1)}=f_{2}, \quad n^{(j)}=(d-1), \quad \sigma^{*(j-1)}=\sigma^{*(j)}=\sigma^{*}
$$

in (11.26). Berryman used this special property to prove that the approximation always lies inside the Beran bounds [18].

With the choice (14.2) of geometric parameters, which is incidentally invariant under changes of reference media, the continued fraction expansion for $\Omega_{a, b}^{(1)}$ implied by (11.13) has exactly the same structure as the continued fraction expansion for $\delta_{a b} \Omega^{(0)}$. Hence we can make the identification

$$
\Omega_{a \ell, b k}^{(1)}=\sigma^{*} \delta_{a b} \delta_{\ell k}, \quad \Omega_{\ell, k}^{(0)}=\sigma^{*} \delta_{\ell k} .
$$

Substituting these in (11.13), and setting $j=1$, yields the self-consistent equation

$$
\sigma^{*}=\sum_{a=1}^{n} f_{a} \sigma_{a}-\sum_{a, b \neq q} \delta \sigma_{a}^{(q)} \widetilde{O}_{a, b} \delta \sigma_{b}^{(q)},
$$

where $\widetilde{O}$ is the inverse of the matrix

$$
O_{a, b}=\left(\sigma_{q}+n^{0} \sigma^{*}\right) / f_{q}+\left(\sigma_{a}+n^{0} \sigma^{*}\right) \delta_{a b} / f_{a},
$$

which is of the form (7.10). Hence, from the expression (7.11) for its inverse, we have

$$
\widetilde{O}_{a, b}=\frac{f_{a} \delta_{a b}}{\sigma_{a}+n^{0} \sigma^{*}}-\frac{f_{a} f_{b}\left[\sum_{c=1}^{n} f_{c} /\left(\sigma_{c}+n^{0} \sigma^{*}\right)\right]^{-1}}{\left(\sigma_{a}+n^{0} \sigma^{*}\right)\left(\sigma_{b}+n^{0} \sigma^{*}\right)} .
$$

Inserting this in (14.6) gives, after some straightforward algebraic manipulation, the result

$$
\begin{aligned}
\left(\sigma^{*}-\sigma_{q}\right) \sum_{c=1}^{n} f_{c} /\left(\sigma_{c}+n^{0} \sigma^{*}\right)= & {\left[\sum_{a=1}^{n} f_{a} \delta \sigma_{a} /\left(\sigma_{a}+n^{0} \sigma^{*}\right)\right] } \\
& \times\left[\sum_{b=1}^{n} f_{b}\left(\sigma_{q}+n^{0} \sigma^{*}+\delta \sigma_{b}\right) /\left(\sigma_{b}+n^{0} \sigma^{*}\right)\right],
\end{aligned}
$$


where the expression between the last square brackets is clearly unity. Thus the formula simplifies and is equivalent to the effective medium approximation, (14.1): the dependence on $\sigma_{q}$ drops out of the equation.

It is striking that the standard effective medium approximation formula corresponds to such a simple choice of fundamental geometric parameters and is a strong indication that these parameters are natural ones for describing the conductivity properties of composites.

One obvious generalization of the approximation (14.2) to anisotropic composites is obtained by taking

$$
W_{a, \lambda, \eta}^{(q)}=f_{a} \delta_{\lambda \eta}, \quad N_{\alpha \ell, \beta \ell}^{(q)}=n_{\ell k}^{0} \delta_{\alpha \beta},
$$

where the matrix $n^{0}$ determines the degree of anisotropy and has non-negative eigenvalues $n_{\ell}^{0}, \ell=1,2, \ldots, d$, satisfying

$$
\sum_{\ell=1}^{d} 1 /\left(n_{\ell}^{0}+1\right)=1
$$

to ensure (8.23) holds. The above set of geometric parameters gives a conductivity matrix $\sigma^{*}$ that commutes with $n^{0}$ and is the solution of the self-consistent equation

$$
\sum_{a=1}^{n} f_{a}\left(\sigma_{a} l-\sigma^{*}\right)\left(\sigma_{a} I+n^{0} \sigma^{*}\right)^{-1}=0
$$

This, in fact, corresponds to the effective medium approximation $[21,59,60]$ for an aggregate of aligned ellipsoids of various conductivities $\sigma_{a}, a=1,2, \ldots, n$. The ellipsoids have a variety of sizes which must extend to the infinitesimal to ensure they can be packed to fill all space. They all have the same eccentricity and the principal axes of each ellipsoid are aligned with the eigenvectors of $n^{0}$. The eigenvalues $n_{\ell}^{0}, \ell=1,2, \ldots, d$, of $n^{0}$ are given in terms of the ellipsoid eccentricity through the relations

$$
n_{\ell}^{0}=\left(1 / L_{\ell}\right)-1
$$

where the constants $L_{\ell}, \ell=1,2, \ldots, d$, are simply the depolarization factors of each ellipsoid along the various axes [61].

\section{Application to Related Transport Problems}

In this section, following earlier work of Dell-Antonio et al. [5], we give examples of the applicability of our results to transport problems aside from the effective electrical and thermal conductivity, dielectric constant, magnetic permeability, and diffusion constant of composites built from isotropic components.

Clearly the whole analysis applies whenever we have a Hilbert space

$$
\mathscr{H}^{\prime}=\mathscr{U} \oplus \mathscr{E}^{\prime} \oplus \mathscr{J}^{\prime}=\mathscr{P}_{1}^{\prime} \oplus \mathscr{P}_{2}^{\prime} \oplus \ldots \oplus \mathscr{P}_{n}^{\prime},
$$

where $\mathscr{U}$ has finite dimension and where the subspaces $\mathscr{U}, \mathscr{E}^{\prime}, \mathscr{J}^{\prime}$, and $\mathscr{P}_{a}^{\prime}$, $a=1,2, \ldots, n$, are real-symmetric with respect to some conjugation $\mathscr{K}$ in $\mathscr{H}:$ a conjugation satisfies

$$
\begin{gathered}
\mathscr{K}\left|a_{1} \mathbf{P}+a_{2} \mathbf{P}^{\prime}\right\rangle=\bar{a}_{1} \mathscr{K}|\mathbf{P}\rangle+\bar{a}_{2} \mathscr{K}\left|\mathbf{P}^{\prime}\right\rangle, \\
\mathscr{K}^{2}=I, \quad\left\langle\mathscr{K} \mathbf{P} \mid \mathscr{K} \mathbf{P}^{\prime}\right\rangle=\left\langle\mathbf{P}^{\prime} \mid \mathbf{P}\right\rangle,
\end{gathered}
$$


for all $\mathbf{P}, \mathbf{P}^{\prime} \in \mathscr{H}$ and for all constants $a_{1}$ and $a_{2}$, with complex conjugates $\bar{a}_{1}$ and $\bar{a}_{2}$ [62]. Actually there is no need to insist upon real-symmetric subspaces. A continued fraction expansion can still be developed in terms of a set of hermitian (rather than symmetric real) weights and normalization factors. Of course, this result is useful only when the field equation (2.11) and the tensor $\sigma^{*}$ have a physical interpretation. In the examples given below, the subspaces $\mathscr{U}, \mathscr{E}^{\prime}, \mathscr{J}^{\prime}$, and $\mathscr{P}_{a}^{\prime}$, $a=1,2, \ldots, n$ are all real-symmetric. Nevertheless, hermitian weights and normalization factors may be needed to describe propagation of radiation in gyrotropic media [61].

Now consider a periodic polycrystalline material comprised of say $j \geqq 1$ types of anisotropic crystallite at varying orientations in the material: different grains of the same type of crystal need not have their principle axes aligned. In each grain we will assume that the local conductivity tensor $\sigma(\mathbf{x})$ is such that $\operatorname{Re} \sigma(\mathbf{x})$ and $\operatorname{Im} \sigma(\mathbf{x})$ share a common set of $d$ (real) eigenvectors. The eigenvectors will, of course, generally change direction across the boundary between two crystallites. Hence as $\mathbf{x}$ is varied over the unit cell $\mathscr{D}$ the eigenvalues of $\sigma(\mathbf{x})$ take $n \leqq j d$ distinct possibly complex values $\sigma_{1}, \sigma_{2}, \ldots, \sigma_{n}$. The Hilbert spaces $\mathscr{U}, \mathscr{E}^{\prime}$, and $\mathscr{J}^{\prime}$ are defined as in Sect. 2. We redefine $\mathscr{P}_{a}, a=1,2, \ldots, n$, as the set of all fields $\mathbf{P} \in \mathscr{H}^{\prime}$ such that

$$
\sigma(\mathbf{x}) \mathbf{P}(\mathbf{x})=\sigma_{a} \mathbf{P}(\mathbf{x})
$$

at all points $\mathbf{x} \in \mathscr{D}$ not on the boundary of a crystallite. Thus $\mathbf{P}(\mathbf{x})$ is locally directed parallel to any eigenvector of $\sigma(\mathbf{x})$ that has eigenvalue $\sigma_{a}$. When the crystallites are isotropic the definition of $\mathscr{P}_{a}^{\prime}$ given here clearly reduces to the one given in Sect. 2.

Thus, in this polycrystalline material the effective conductivity tensor $\sigma^{*}$ is an $\Omega$ function of the eigenvalues $\sigma_{1}, \sigma_{2}, \ldots, \sigma_{n}$ of the local conductivity tensor. All the bounds derived in Part II extend to $\sigma^{*}$. Note, however, that the elementary weights and normalization factors need not satisfy the rather special equations (8.22) and (8.23).

A similar argument shows the results apply to the effective viscoelasticity tensors of polycrystalline media. As is customary [63], we treat the local viscoelastic tensor as a $d^{0} \times d^{0}$ matrix, $\sigma(\mathbf{x})$, where $d^{0} \equiv \frac{1}{2} d(d+1)$ and $d$ is the dimensionality of the composite. We assume that $\operatorname{Re} \sigma(\mathbf{x})$ and $\operatorname{Im} \sigma(\mathbf{x})$ share a local common set of eigenvectors and remain constant throughout each crystallite. The set of eigenvalues, $\sigma_{1}, \sigma_{2}, \ldots, \sigma_{n}$, of the local viscoelastic tensor take real or complex values. The space $\mathscr{U}$ of uniform strain (or stress) fields has dimension $d^{0}$, and we define $\mathscr{E}^{\prime}$ (or $\mathscr{J}^{\prime}$ ) to consist of those periodic square-integrable strain (or stress) fields that have zero average value over the unit cell $\mathscr{D}$. Each component Hilbert space $\mathscr{P}_{a}^{\prime}, a=1,2, \ldots, n$, consists of the elastic fields $\mathbf{P}(\mathbf{x})$ satisfying $(15.2)$ for all $\mathbf{x} \in \mathscr{D}$. With these definitions the effective viscoelasticity tensor $\sigma^{*}$ is clearly a rank $\frac{1}{2} d(d+1) \Omega$-function of the eigenvalues of the local viscoelasticity tensor.

More generally we can consider non-local linear conductivity tensors and nonlocal linear viscoelasticity tensors. The non-local operator $\sigma$ must, however, have a finite number of eigenvalues $\sigma_{a}, a=1,2, \ldots, n$, and the eigenfunctions corresponding to each eigenvalue $\sigma_{a}$ must span some real-symmetric Hilbert space of fields, defined as $\mathscr{P}_{a}^{\prime}$.

The mathematical framework also applies to multiterminal, multicomponent linear impedance networks, consisting of $d+1$ terminals connected to a net- 
work of $h$ impedances that have various types of complex conductances $\sigma_{1}(\omega), \sigma_{2}(\omega), \ldots, \sigma_{n}(\omega)$ as a function of the frequency $\omega$. (Some of the impedances may share the same conductance and so we have $n \leqq h$.) Let us label the impedances by an index $i=1,2, \ldots, h$ and arbitrarily assign a + sign to one side of each impedance while tagging the opposite side with a - sign. Any field in the network is represented by a vector $\mathbf{P}$ with complex components $P_{i}, i=1,2, \ldots, h$, representing the directed field in impedance $i$. We let $\mathscr{H}^{\prime}$ denote the $h$-dimensional space comprised of all such fields and in $\mathscr{H}^{\prime}$ we define the standard inner product

$$
\left\langle\mathbf{P} \mid \mathbf{P}^{\prime}\right\rangle=\sum_{i=1}^{h} \bar{P}_{i} P_{i}^{\prime}
$$

for any two fields $\mathbf{P}, \mathbf{P}^{\prime} \in \mathscr{H}$, where the bar denotes complex conjugation.

The impedances are joined at a set of say $k+1$ nodes. Some of these nodes represent the $d+1$ terminals. We let an index $j=0,1,2, \ldots, k$ label the nodes, starting with the terminals. Thus the terminals are labelled by indices $j \leqq d$. The geometry of the network is represented by the connection coefficients $r_{i, j}$ defined by

$$
\begin{aligned}
r_{i, j} & = \pm 1 & & \text { if the } \pm \text { side of impedance } i \text { is connected to node } j \\
& =0 & & \text { otherwise. }
\end{aligned}
$$

Each row, $i$, of this $h \times(k+1)$ matrix $r_{i, j}$ contains only two non-zero elements, +1 and -1 , signifying the two nodes connected by impedance $i$.

The $(k-d)$-dimensional subspace $\mathscr{E}^{\prime}$ consists of those fields $\mathbf{E} \in \mathscr{H}^{\prime}$ satisfying

$$
E_{i}=\sum_{j=0}^{k} r_{i, j} \phi_{j}
$$

for some complex potential $\phi_{j}$ such that

$$
\phi_{j}=0 \text { for all } j \leqq d .
$$

The $(h-k)$-dimensional subspace $\mathscr{J}^{\prime}$ consists of fields $\mathbf{J} \in \mathscr{H}^{\prime}$ satisfying

$$
\sum_{i=1}^{h} r_{i, j} J_{i}=0 \text { for all } j,
$$

while the $d$-dimensional subspace $\mathscr{U}$ is comprised of fields $\mathbf{e} \in \mathscr{H}^{\prime}$ of the form (15.4) satisfying the additional constraint that

$$
\sum_{i=1}^{h} r_{i, j} e_{i}=0 \text { for all } j>d,
$$

where the associated potential $\phi_{k}$ need not be compatible with the boundary conditions (15.5). The three subspaces $\mathscr{U}, \mathscr{E}$, and $\mathscr{J}^{\prime}$ are clearly orthogonal, and by adding their dimensions we deduce that they span $\mathscr{H}^{\prime}$.

Each subspace $\mathscr{P}_{a}^{\prime}$ consists of those fields $\mathbf{P}$ such that $P_{i}$ is non-zero only in the impedances $i$ with complex conductance $\sigma_{a}$. In the Hilbert space $\mathscr{H}^{\prime}$ thus defined the effective tensor $\sigma^{*}$ gives the electrical response of the network (measured in terms of voltages and currents at the terminals) relative to the response when $\sigma_{a}=1$ for all $a$. Since $\mathscr{H}^{\prime}$ has finite dimension the $\Omega$-function $\sigma^{*}\left(\sigma_{1}, \sigma_{2}, \ldots, \sigma_{n}\right)$ must be a terminating $\Omega$-function. The weights and normalization factors thus provide a 
characterization of the analytic functions that result from multiterminal, multicomponent impedance networks. This may have practical applications to the design and synthesis of electrical networks [64].

\section{Appendix 1: Simplified Definitions of the Canonical Basis Set of Fields and the Weight and Normalization Matrices}

The definitions given in Sect. 8 of the canonical basis set of fields and the weight and normalization matrices are awkward because they entail the introduction of several intermediate basis sets of fields. Here we give simpler and more direct inductive definitions that help shed light on the physical significance of these quantities.

Given any basis set of orthonormal fields $\mathbf{x}_{\ell}, \ell=1,2, \ldots, d$, that spans $\mathscr{U}$ we set $\mathbf{x}_{\ell}^{(q)}=\mathbf{x}_{\ell}$. Now suppose that for some $j$ the fields $\mathbf{x}_{\omega}^{(q)}$ have been defined for all strings $\omega$ with $o(\omega)=j$ : this is clearly true for $j=0$. The associated weight matrices $W_{a}^{(j)}$ can be defined via the identity

$$
W_{a, \omega, \eta}^{(q)} \equiv\left\langle\mathbf{x}_{\omega}^{(q)}\left|\chi_{a}\right| \mathbf{x}_{\eta}^{(q)}\right\rangle,
$$

where $o(\omega)=O(\eta)=j$. The auxiliary fields

$$
\left|\mathbf{a}_{a \omega}^{(q)}\right\rangle \equiv \chi_{a}\left|\mathbf{x}_{\omega}^{(q)}\right\rangle-W_{a, \omega, \eta}^{(q)}\left|\mathbf{x}_{\eta}^{(q)}\right\rangle
$$

are then of higher order, $j+1$, and orthogonal to the fields $\mathbf{x}_{\tau}^{(q)}$ with $o(\tau)=j$. They are not however normalized. From (A1.1) and (A1.2) their inner products are given by

$$
\left\langle\mathbf{a}_{a \omega}^{(q)} \mid \mathbf{a}_{b \tau}^{(q)}\right\rangle=\delta_{a b} W_{a, \omega, \tau}^{(q)}-W_{a, \omega, \eta}^{(q)} W_{b, \eta, \tau}^{(q)} \equiv Y_{a \omega, b \tau}^{(q)},
$$

and so to obtain the next set of orthonormal fields in the canonical basis set we take

$$
\left|\mathbf{y}_{a \omega}^{(q)}\right\rangle \equiv \dot{Y}_{a \omega, b \tau}^{(q)}\left|a_{b \tau}^{(q)}\right\rangle=\dot{Y}_{a \omega, b \tau}^{(q)}\left(\chi_{b}\left|\mathbf{x}_{\tau}^{(q)}\right\rangle-W_{b, \tau, \eta}^{(q)}\left|\mathbf{x}_{\eta}^{(q)}\right\rangle\right)
$$

Similarly starting from these fields, we define the matrices

$$
\begin{aligned}
U_{a \omega, b \tau}^{\prime(q)} & \equiv\left\langle\mathbf{y}_{a \omega}^{(q)}\left|\Gamma_{1}\right| \mathbf{y}_{b \tau}^{(q)}\right\rangle, \\
V_{a \omega, b \tau}^{(q)} & \equiv\left\langle\mathbf{y}_{a \omega}^{(q)}\left|\Gamma_{2}\right| \mathbf{y}_{b \tau}^{(q)}\right\rangle=\delta_{a \omega, b \tau}-U_{a \omega, b \tau}^{(q)},
\end{aligned}
$$

and we introduce the set of auxiliary fields

$$
\left|\mathbf{b}_{a \omega}^{(q)}\right\rangle \equiv \Gamma_{1}\left|\mathbf{y}_{a \omega}^{(q)}\right\rangle-\sum_{b \neq q} U_{a \omega, b \tau}^{(q)}\left|\mathbf{y}_{b \tau}^{(q)}\right\rangle,
$$

which are orthogonal to the fields $\left|y_{c \eta}^{(q)}\right\rangle$ with $o(\eta)=j$ and have inner products

$$
\left\langle\mathbf{b}_{a \omega}^{(q)} \mid \mathbf{b}_{b \tau}^{(q)}\right\rangle=\sum_{c \neq q} U_{a \omega, c \eta}^{\prime(q)} V_{c \eta, b \tau}^{\prime(q)} .
$$

By normalizing the auxiliary fields we subsequently obtain the set of fields

$$
\left|\mathbf{x}_{a \omega}^{(q)}\right\rangle=\sum_{c, d \neq q} \dot{U}_{a \omega, c \eta}^{\prime(q)} \dot{V}_{c \eta, d \tau}^{\prime(q)}\left(\Gamma_{1}\left|\mathbf{y}_{d \tau}^{(q)}\right\rangle-\sum_{b \neq q} U_{d \tau, b \lambda}^{\prime(q)}\left|\mathbf{y}_{b \lambda}^{(q)}\right\rangle\right)
$$

at the next level in the hierarchy of the canonical basis set. 
The relations (A1.1), (A1.4), (A1.5), and (A1.8) clearly serve to define by induction the weights and the canonical basis set of fields. The normalization matrices are defined via (8.3) in terms of the matrix $U^{\prime}$ that is obtained from (A1.5).

\section{Appendix 2: Continued Fraction Expansion from Hashin-Shtrikman Variational Principles}

Here we show how the continued fraction expansion for $\sigma^{*}$ implied by (12.2), (12.3), (12.7), and (11.12) can alternatively be derived on the basis of the HashinShtrikman variational formulation [19] for $\sigma^{*}$,

$$
\left\langle\mathbf{e} \mid \sigma^{*}-\sigma_{q} / \mathbf{e}\right\rangle=\min _{P \in \mathscr{H}}\left[2\langle\mathbf{e} \mid \mathbf{P}\rangle-\sigma_{q}^{-1}\left\langle\mathbf{P}\left|\Gamma_{1}\right| \mathbf{P}\right\rangle+\sum_{a=1}^{n} \sigma_{q}^{-1} s_{a}^{(q)}\left\langle\mathbf{P}\left|\chi_{a}\right| \mathbf{P}\right\rangle\right],
$$

which holds whenever $\sigma_{q} \geqq \sigma_{a}$ for all components $\sigma_{a}$. If $\sigma_{q} \leqq \sigma_{a}$ for all $\sigma_{a}$, then the variational principle still applies provided the minimum over $\mathbf{P} \in \mathscr{H}$ in (A2.1) is replaced by the maximum over $\mathbf{P} \in \mathscr{H}$.

Since the minimum in (A2.1) is attained within the Hilbert space spanned by the polarization fields $\mathbf{P}_{a \tau}^{(q)}$, with $a \neq q$, we only need consider fields of the form

$$
\mathbf{P}=\sum_{a \neq q} \alpha_{a \tau} \mathbf{P}_{a \tau}^{(q)},
$$

which when substituted in the variational principle (A2.1) gives

$$
\mathbf{e}^{T} \sigma^{*} \mathbf{e}=\sigma_{q} \mathbf{e}^{T} \mathbf{e}+\min _{\alpha}\left[\sum_{a \neq q} 2 \mathbf{e}^{T} Z_{a} \boldsymbol{\alpha}_{a}+\sigma_{q}^{-1} \sum_{a, b \neq q} \boldsymbol{\alpha}_{a}^{T}\left(s_{a}^{(q)} \delta_{a b} Z_{a}-Z_{1, a, b}^{\dagger}\right) \boldsymbol{\alpha}_{b}\right] .
$$

By defining $\boldsymbol{\alpha}_{a}^{(j+1)}, Z_{a}^{(j)}$, and $Z_{1, a, b}^{\dagger(j+1)}$ as those subvectors and submatrices of $\boldsymbol{\alpha}_{a}, Z_{a}$, and $Z_{1, a, b}^{\dagger}$ that have elements $\alpha_{a \omega}, Z_{a, \omega, \tau}^{(q)}$, and $Z_{1, a \omega, b \tau}^{\dagger(q)}$ with $o(\omega)=o(\tau)=j$, we obtain, via (6.12), the formula

$$
\begin{aligned}
\mathbf{e}^{T} \sigma^{*} \mathbf{e}= & \sigma_{q} \mathbf{e}^{T} \mathbf{e}+\min _{\alpha} \lim _{h \rightarrow \infty}\left[\sum_{a \neq q} 2 \mathbf{e}^{T} Z_{a}^{(0)} \boldsymbol{\alpha}_{a}^{(1)}\right. \\
& \left.+\sigma_{q}^{-1} \sum_{a, b \neq q}\left(\sum_{j=1}^{h} \boldsymbol{\alpha}_{a}^{(j) T} \Xi_{a, b}^{(j, j)} \boldsymbol{\alpha}_{b}^{(j)}+\sum_{j=2}^{h} \boldsymbol{\alpha}_{a}^{(j-1) T} Z_{b, a}^{(j-1)} \boldsymbol{\alpha}_{b}^{(j)}\right)\right],
\end{aligned}
$$

in which

$$
\Xi_{a, b}^{(j, j)} \equiv \delta_{a b} s_{a}^{(a)} Z_{a}^{(j-1)}-Z_{1, a, b}^{\dagger(j)} .
$$

We assume the minimum over $\alpha$ and the limit $h \rightarrow \infty$ in (A2.4) can be interchanged. The best choice of $\boldsymbol{\alpha}_{a}^{(h)}$ in the square bracketed expression in (A2.4) is then obviously

$$
\boldsymbol{\alpha}_{a}^{(h)}=-\sum_{b, c \neq q} \tilde{\Xi}_{a, b}^{(h, h)} Z_{b, c}^{(h-1) T} \boldsymbol{\alpha}_{c}^{(h-1)},
$$

where $\cong^{(h, h)}$ is the inverse of $\Xi^{(h, h)}$. This suggests, when (A2.6) is substituted in (A2.4), that we should define the hierarchy of matrices

$$
\Xi^{(h, j)} \equiv \Xi^{(j, j)}-\sum_{x, y \neq q} Z_{x}^{(j)} \stackrel{\cong}{\tilde{\Xi}_{x, y}^{(h, j+1)}} Z_{y}^{(j)},
$$


for $j=h-1, h-2, \ldots, 1$. By iterating the optimization procedure, redefining

$$
\Omega^{(0)}=\sigma_{q} I-\sigma_{q} \sum_{a, b \neq q} Z_{a}^{(0)} \stackrel{\underset{\Xi}{=}(h, 1)}{=} Z_{b}^{(0)},
$$

and taking the limit $h \rightarrow \infty$, the result

$$
\sigma^{*}=\Omega^{(0)} \quad(h \rightarrow \infty)
$$

is finally obtained. When $h$ is kept finite (A2.9) is replaced by the bounds

$$
\begin{array}{lll}
\sigma^{*} \leqq \Omega^{(0)} & \text { when } \quad \sigma_{q} \geqq \sigma_{a} & \forall a, \\
\sigma^{*} \geqq \Omega^{(0)} & \text { when } \quad \sigma_{q} \leqq \sigma_{a} & \forall a .
\end{array}
$$

To express the continued fraction that results from (A2.8) and (A2.7) in terms of the fundamental geometric parameters, let us introduce, for $j \leqq h-1$, the matrices

$$
\begin{aligned}
\Delta_{a, b}^{+(1)} & =-\dot{W}_{a}^{(0)} \Xi_{a, b)}^{(h, 1)} \dot{W}_{b}^{(0)}, \\
\Delta_{a, b}^{+(j+1)} & =-\dot{W}_{a}^{(j)} K^{(j)} \Xi_{a, b}^{(h, j+1)} K^{(j) T} \dot{W}_{b}^{(j)}-\hat{W}_{a}^{(j)} \tilde{K}^{(j) T} \widetilde{V}^{(j)} \widetilde{K}^{(j)} \hat{W}_{b}^{(j)}, \\
\tilde{\Pi}^{+(j)} & =\widehat{V}^{(j)}\left[I^{(j)}+\sum_{x, y \neq q} \widehat{O}^{(j)} W_{x}^{(j)} \widetilde{K}_{x}^{(j) T} \underline{\Xi}_{x, y}^{(h, j)} \tilde{K}_{y}^{(j)} W_{y}^{(j)} \hat{U}^{(j)}\right] \widehat{V}^{(j)}, \\
\tilde{\Pi}^{+(h)} & =V^{(h)}=\left(I+N^{(h)}\right)^{-1},
\end{aligned}
$$

which remain to be shown equivalent to the conductivity matrices $\Delta^{+}$and $\tilde{\Pi}^{+}$ defined in Sect. 12, for $\Omega^{(h)}$ of the form (12.25) with $\sigma_{m}=\sigma_{q}$.

First note that (A2.9) with (A2.8) directly implies the formula

$$
\Omega^{(0)}=\sigma_{q} I-\sigma_{q} \sum_{a, b \neq q} \hat{W}_{a}^{(0)} \tilde{\Delta}_{a, b}^{(1)} \hat{W}_{b}^{(0)},
$$

which is consistent with (12.3). Next by substituting (A2.7) in (A2.11) and using (A2.5) with the expressions (6.10) and (6.11) for the matrix $Z_{1}^{+}$we deduce that

$$
\Delta_{a, b}^{+(j)}=-S_{a} I_{a, b}^{(j)}+\dot{W}_{a}^{(j-1)} K^{(j-1)}\left[V_{a, b}^{(j)}+\sum_{x, y \neq q} Z_{x, a}^{(j)} \stackrel{\stackrel{\Xi}{\Xi}_{x, y}^{(h, j+1)}}{Z_{y, b}^{(j) T}}\right] K^{(j-1) T} \dot{W}_{b}^{(j-1)} .
$$

This expression with (8.28) and (8.29) clearly yields the recursion relation (12.7) which now links $\Delta^{+}$defined by (A2.11) with $\Pi^{+}$defined by (A2.12).

Lastly, since the expression (A2.12) for $\tilde{\Pi}^{+}$is of the form (12.15), its inverse can be readily calculated, via (12.16), and for $j<h$ we find

$$
\Pi^{+(j)}=I^{(j)}+\hat{N}^{(j)}\left[I^{(j)}+\sum_{x, y \neq q} \hat{W}_{x}^{(j)} \tilde{\Delta}_{x, y}^{+(j+1)} \hat{W}_{y}^{(j)}\right] \hat{N}^{(j)}
$$

This with (12.7) and (A2.13) clearly implies a continued fraction for $\Omega^{(0)}$ that is identical to the continued fraction resulting from (12.2), (12.3), (12.7), and (11.12) and enables us to identify the matrices $\Pi^{+}$and $\Delta^{+}$defined through (A2.9)-(A2.11) with the matrices defined in Sect. 12, when $\Omega^{(h)}$ has the form (12.25) with $\sigma_{m}=\sigma_{q}$. 
Prof. M. Berry, Dr. J. G. Berryman, Prof. M. C. Cross, Prof. M. E. Fisher, Dr. P. Graves-Morris, Prof. S. E. Koonin, and Prof. G. Papanicolaou. The support of the California Institute of Technology, through a Weingart Fellowship, and the support of Chevron Laboratories, through a research grant, is much appreciated. In addition I am grateful to the National Science Foundation for partial support during my stay at the Aspen Center for Physics, where a significant portion of this work was completed.

\section{References}

1. Wall, H.S.: Analytic theory of continued fractions. New York: Van Nostrand 1948

2. Jones, W.B., Thron, W.J.: Encyclopedia of mathematics and its applications, Vol. 11. Rota, G.-C. (ed.). London: Addison-Wesley 1980

3. Baker, G.A., Jr., Graves-Morris, P.R.: Encyclopedia of mathematics and its applications, Vols. 13 and 14. Rota, G.-C. (ed.). London: Addison-Wesley 1981

4. Batchelor, G.K.: Transport properties of two-phase materials with random structure. Ann. Rev. Fluid. Mech. 6, 227 (1974)

5. Dell'Antonio, G.F., Figari, R., Orlandi, E.: An approach through orthogonal projections to the study of inhomogeneous or random media with linear response. Ann. Inst. Henri Poincaré 44, 1 (1986)

6. Doyle, W.T.: The Clausius-Mossotti problem for cubic arrays of spheres. J. Appl. Phys. 49, $795(1978)$

7. McKenzie, D.R., McPhedran, R.C., Derrick, G.H.: The conductivity of lattices of spheres. II. The body centered and face centered cubic lattices. Proc. R. Soc. Lond. A 362, 211 (1978)

8. Perrins, W.T., McKenzie, D.R., McPhedran, R.C.: Transport properties of regular arrays of cylinders. Proc. R. Soc. Lond. A 369, 207 (1979)

9. Sangani, A.S., Acrivos, A.: On the effective thermal conductivity and permeability of regular arrays of spheres. In: Macroscopic properties of disordered media, p. 216. Burridge, R., Childress, S., Papanicolaou, G. (eds.). Berlin, Heidelberg, New York: Springer 1982

10. Bessis, D., Villani, M.: Perturbative-variational approximations to the spectral properties of semibounded Hilbert space operators, based on the moment problem with finite or diverging moments. Application to quantum mechanical systems. J. Math. Phys. 16, 462 (1975)

11. Bergman, D.J.: The dielectric constant of a composite material - a problem in classical physics. Phys. Rep. C 43, 377 (1978)

12. Milton, G.W., Golden, K.: Thermal conduction in composites. In: Thermal conductivity 18 , Ashworth, T., Smith, D.R. (eds.). New York: Plenum Press 1985

13. Milton, G.W.: Modelling the properties of composites by laminates. In: Homogenization and effective moduli of materials and media, p. 150. Ericksen, J.L., Kinderlehrer, D., Kohn, R., Lions, J.-L. (eds.). Berlin, Heidelberg, New York: Springer 1986

14. Golden, K.: Bounds on the complex permittivity of a multicomponent material. J. Mech. Phys. Solids (to appear)

15. Bergman, D.J.: Rigorous bounds for the complex dielectric constant of a two-component composite. Ann. Phys. 138, 78 (1982)

16. Golden, K., Papanicolaou, G.: Bounds for effective parameters of multicomponent media by analytic continuation. J. Stat. Phys. 40, 655 (1985)

17. Wiener, O.: Abhandlungen der Mathematisch-Physischen Klasse der Königlichen Sächsischen Gesellschaft der Wissenschaften 32, 509 (1912)

18. Beran, M.J.: Use of the variational approach to determine bounds for the effective permittivity in random media. Nuovo Cimento 38, 771 (1965)

19. Hashin, Z., Shtrikman, S.: A variational approach to the theory of the effective magnetic permeability of multiphase materials. J. Appl. Phys. 33, 3125 (1962)

20. Kröner, E.: Bounds for effective elastic moduli of disordered materials. J. Mech. Phys. Solids 25, 137 (1977)

21. Willis, J.R.: Elasticity theory of composites. In: Mechanics of solids, p. 653. Hopkins, H.G., Sewell, M.J. (eds.). Oxford, New York: Pergamon Press 1982 
22. Phan-Thien, N., Milton, G.W.: New bounds on the effective thermal conductivity of $N$-phase materials. Proc. R. Soc. London A 380, 333 (1982)

23. Bruggeman, D.A.G.: Berechnung verschiedener physikalischer Konstanten von heterogen Substanzen. I. Dielektrizitätskonstanten und Leitfähigkeiten der Mischkörper aus isotropen Substanzen. Ann. Phys. (Leipzig) 24, 636 (1935)

24. Kohler, W., Papanicolaou, G.C.: Bounds for effective conductivity of random media. In: Macroscopic properties of disordered media, p. 111. Burridge, R., Childress, S., Papanicolaou, G. (eds.). Berlin, Heidelberg, New York: Springer 1982

25. Kantor, Y., Bergman, D.J.: Improved rigorous bounds on the effective elastic moduli of a composite material. J. Mech. Phys. Solids 32, 41 (1984)

26. Papanicolaou, G.C., Varadan, S.R.S.: Boundary value problems with rapidly oscillating random coefficients. In: Colloquia mathematica societatis jános boyai 27, random fields, Esztergom (Hungary) 1982, p. 835. Amsterdam: North-Holland

27. Golden, K., Papanicolaou, G.: Bounds for effective parameters of heterogeneous media by analytic continuation. Commun. Math. Phys. 90, 473 (1983)

28. Gilbarg, N., Trudinger, N.S.: Elliptic partial differential equations of second order, p. 78. Berlin, Heidelberg, New York: Springer 1977

29. Brown, W.F.: Solid mixture permittivities. J. Chem. Phys. 23, 1514 (1955)

30. Torquato, S.: Effective electrical conductivity of two-phase disordered composite media. J. Appl. Phys. 58, 3790 (1985)

31. Beran, M.J.: Statistical continuum theories, pp. 181-256. New York: Interscience 1968

32. Corson, P.B.: Correlation functions for predicting properties of heterogeneous materials. I. Experimental measurement of spatial correlation functions in multiphase solids. J. Appl. Phys. 45, 3159 (1974)

33. Torquato, S., Stell, G.: Microstructure of two-phase random media. I. The n-point probability functions. J. Chem. Phys. 77, 2071 (1982)

34. Gillette, G.J.: Effective property bounds for two-phase random suspensions using statistics of inclusion geometry. Thesis, Catholic University of America, Washington, D.C., 1984

35. Berryman, J.G.: Measurement of spatial correlation functions using image processing techniques. J. Appl. Phys. 57, 2374 (1985)

36. Haile, J.M., Massobrio, C., Torquato, S.: Two-point matrix probability function for twophase random media: Computer simulations results for impenetrable spheres. J. Chem. Phys. 83, 4075 (1985)

37. Milton, G.W., Kohn, R.V.: Bounds for anisotropic composites by variational principles (in preparation)

38. Keller, J.B.: A theorem on the conductivity of a composite medium. J. Math. Phys. 5, 548 (1964)

39. Dykhne, A.M.: Conductivity of a two-dimensional, two-phase system. Zh. Eksp. Teor. Fiz. 59, 110 (1970) [Soviet Phys. JETP 32, 63 (1971)]

40. Mendelson, K.S.: Effective conductivity of two-phase material with cylindrical phase boundaries. J. Appl. Phys. 46, 917 (1975)

41. Golden, K.: Bounds for effective parameters of multicomponent media by analytic continuation. Ph.D. thesis: New York University 1984

42. Synge, J.L.: The hypercircle in mathematical physics. London: Cambridge University Press 1957

43. Milton, G.W.: Bounds on the electromagnetic, elastic, and other properties of two-component composites. Phys. Rev. Lett. 46, 542 (1981)

44. Miller, M.N.: Bounds for effective electrical, thermal, and magnetic properties of heterogeneous materials. J. Math. Phys. 10, 1988 (1969)

45. McPhedran, R.C., Milton, G.W.: Bounds and exact theories for the transport properties of inhomogeneous media. Appl. Phys. A 26, 207 (1981)

46. Torquato, S., Stell, G.: Bounds on the effective thermal conductivity of a dispersion of fully penetrable spheres. Lett. Appl. Engng. Sci. 23, 375 (1985)

47. Torquato, S., Beasley, J.D.: Effective properties of fibre-reinforced materials. I. Bounds on the effective thermal conductivity of dispersions of fully penetrable cylinders. Int. J. Engng. Sci. 24, 415 (1986) 
48. Berryman, J.G.: Variational bounds on elastic constants for the penetrable sphere model. J. Phys. D 18, 585 (1985)

49. Felderhof, B.U.: Bounds for the complex dielectric constant of a two-phase composite. Physica 126 A, 430 (1984)

50. Torquato, S., Lado, F.: Effective properties of two-phase disordered composite media. II. Evaluation of bounds on the conductivity and bulk modulus of dispersions of impenetrable spheres. Phys. Rev. B 33, 6428 (1986)

51. Torquato, S., Stell, G.: Microscopic approach to transport in two-phase random media. CEAS Report No. 352, 1980

52. Berryman, J.: Effective medium theory for elastic composites. In: Elastic wave scattering and propagation, p. 111. Varadan, V.K., Varadan, V.V. (eds.). Ann Arbor, MI: Ann Arbor Science 1982

53. Milton, G.W.: The coherent potential approximation is a realizable effective medium scheme. Commun. Math. Phys. 99, 463 (1985)

54. Sen, P.N., Scala, C., Cohen, M.H.: A self-similar model for sedimentary rocks with application to the dielectric constant of fused glass beads. Geophysics 14, 541 (1976)

55. Landauer, R.: Electrical conductivity in inhomogeneous media. In: Electrical transport and optical properties of inhomogeneous media, p. 2. Garland, J.C., Tanner, D.B. (eds.). New York: Am. Inst. Phys. 1978

56. Watt, J.P., Davies, G.F., O'Connell, R.J.: The elastic properties of composite materials. Rev. Geophys. Space Phys. 14, 541 (1976)

57. Korringa, J., Brown, R.J.S., Thompson, D.D., Runge, R.J.: Self-consistent imbedding and the ellipsoidal model for porous rocks. J. Geophys. Res. 84, 5591 (1979)

58. Berryman, J.G.: Long-wavelength propagation in composite elastic media. I. Spherical inclusions. J. Acoust. Soc. Am. 68, 1809 (1980)

59. Niklasson, G.A., Granqvist, C.G.: Optical properties and solar selectivity of coevaporated $\mathrm{Co}-\mathrm{Al}_{2} \mathrm{O}_{3}$ films. J. Appl. Phys. 55, 3382 (1984)

60. Stroud, D.: Generalized effective-medium approach to the conductivity of an inhomogeneous material. Phys. Rev. B 12, 3368 (1975)

61. Landau, L.D., Lifshitz, E.M.: Electrodynamics of continuous media. Oxford, New York: Pergamon Press 1960

62. Weidmann, J.: Linear operators in Hilbert spaces, p. 235. Berlin, Heidelberg, New York: Springer 1980

63. Love, A.E.H.: A treatise on the mathematical theory of elasticity. New York: Dover 1944

64. Storer, J.E.: Passive network synthesis. New York: McGraw-Hill 1957

Communicated by M. E. Fisher

Received February 1, 1986; in revised form November 18, 1986 
